\title{
The Origins of Bosons and Fermions
}

\author{
ShaoXu Ren \\ Institute of physical science and Engineering \\ Tongji University, 200092, Shanghai, China \\ Corresponding email: shaoxu-ren@hotmail.com
}

Received 9 September 2014; revised 24 September 2014; accepted 29 September 2014

\begin{abstract}
This paper proposes that all Bosons and all Fermions originate from even more elementary constituents, which called Spin Angular Momentum Vacuum ( SAMV).

SAMV is filled with Primitive Spin Particles (PSP). The total square spin angular momentum of each PSP is negative, less than zero.

Those PSP labeled by index $-\frac{1}{2}$ of Casimir Operator, are called Vacuum Spin Particle ( VSP ), which could be contracted into so-called Vacuum Bubbles (VB).

VB are identical bubbles, are "sub-observable physical quantities".

VB are paired up into Vacuum Bubble Pair VBP.

VSP $\omega_{j}\left(\right.$ or $\left.\omega^{+}, \omega^{-}\right)$results from Self-identical vacuum bubble interaction $\omega_{\mathrm{S}=0}^{+,-}(k)$ through the zero order Phase Transition PT.

When the 1st, 2nd, 3rd,... order PT of VBP occur, then VBP turn into Bosons and Fermions, excited out of sea level of SAMV ocean.
\end{abstract}

\section{Keywords}

Spin Angular Momentum Vacuum SAMV; Primitive Spin Particles PSP ;

Vacuum Spin Particles ( VSP );Abnormal Casimir Operator ACO ;

Vacuum Contractions VC; Vacuum Bubbles VB ; Vacuum Bubble Pair VBP ;

Phase Transitions PT ; Bosons, Fermions ;

The Third Kind Of Particles TKP ; Chaos Spin Hierarchy CSH ;

The Equivalence of Vacuum Bubbles;

Locality and Nonlocality of Vacuum Contractions;

Local and Nonlocal Angular Momentum Commutations

\section{Introduction}

Why in universe there exist those particles, Bosons with integer value spin $0 \hbar, 1 \hbar, 2 \hbar, \ldots$ and Fermions with half-integer spin $\hbar / 2,3 \hbar / 2,5 \hbar / 2 \ldots$ ?

Where they come from? 
There are all types of Bosons and Fermions in nature, whatever happen with them in any physical interaction processes as known yet, these particles bear themselves quite different, but always keep their spin values to be either integers or half-integers !

The phenomenons show there must be something abstruse, abstruse philosophy.

Spin and energy are fundamental physical quantities used to describe the behaviour of particles. The difference between physical dimension of spin and that of energy is only the frequency factor. Contrary to energy amounts of a particle, always be variable, the spin angular momentum values of a particle are very limited strictly. It seems angular momentum may be more fundamental than energy.

When appreciating the mathematical elegance of "The Third Kind Of Particles", TKP, be struck by an idea that:

Analog of an electron, which could possess its negative energy sea introduced by Dirac's intuitive feelings cite: [1], why not a spin particle possesses its negative angular momentum ocean ? and see what happen.

The values of spin angular momentum values of a particle, maybe too, variable from positive region to negative region, angular momentum and energy are in common in front of physical picture.

Unfortunately, conventional quantum mechanics could not provide a math frame of angular momentum, that can construcct a total square angular momentum with negative eigenvulues.

That's all due to finite demensional and Hermitian matrix representation in conventional quantum mechanics.

That's all due to total square angular momentum operator is always a positive Casimir operator in conventional quantum mechanics.

If strive for mathematical elegance balance between angular momentum and energy, perhaps, the enlightments from Dirac could give us some suggestions.

Turn to TKP, TKP can offer "Abnormal Casimir Operator, ACO ", whose labels of irreducible representation of Lie group with the index of negative values.

CSH and ACO may unveil the mysterious origins of Bosons and Fermions. 


\section{Chaos Spin Hierarchy CSH}

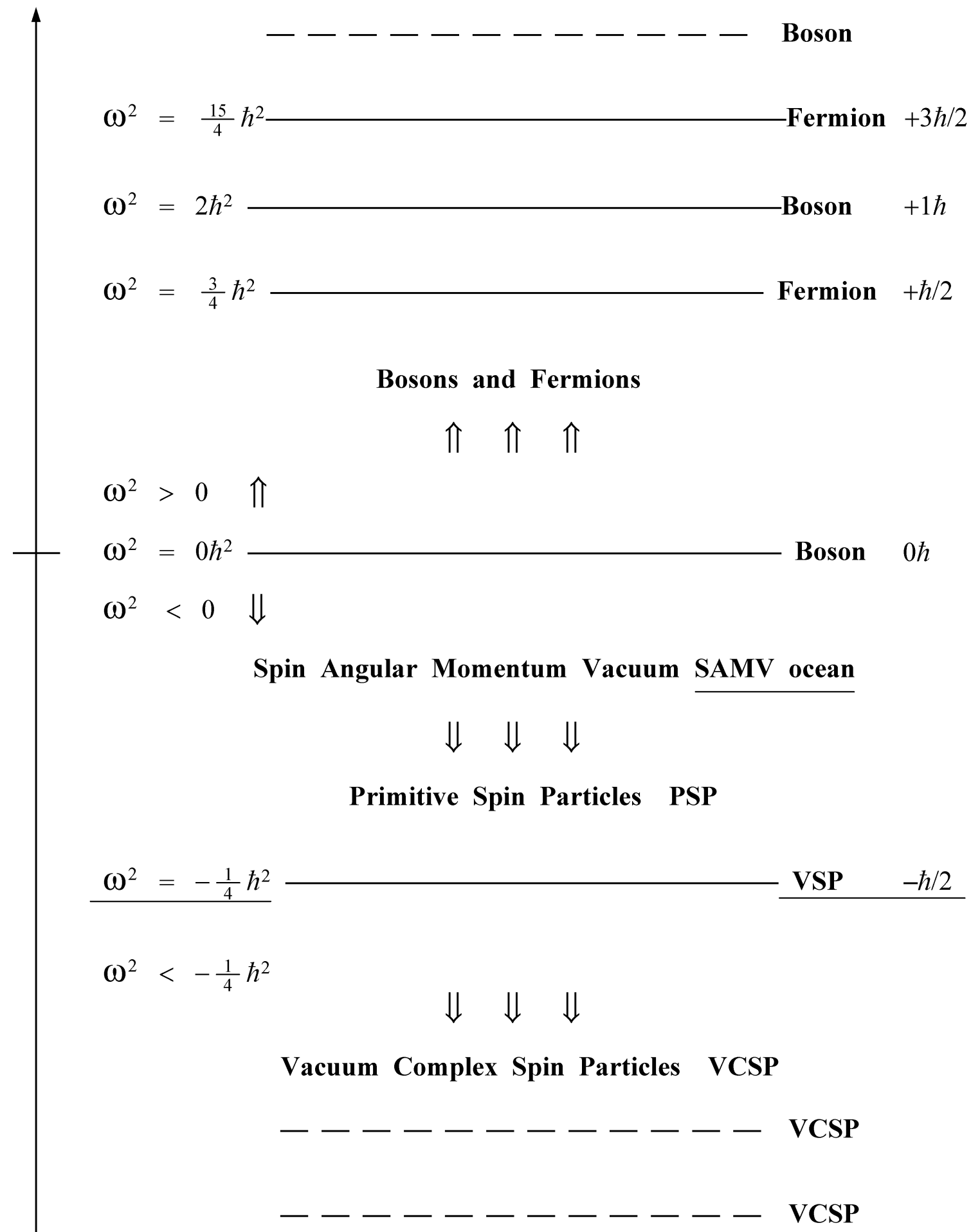

FIG.1. CSH, PSP, VSP and Bosons, Fermions $\omega^{2}$ : Casimir Operator, Vacuum Spin Particles VSP 


\section{Bosons and Fermions in TKP}

Angular momentum commutation rules are given as

$$
\Omega_{i} \Omega_{j}-\Omega_{i} \Omega_{j}=i \hbar \Omega_{k},
$$

$i, j, k=1,2,3$ are circulative. Or by raising operator and lowing operator

$$
\begin{aligned}
& \Omega^{+}=\Omega_{1}+i \Omega_{2} \\
& \Omega^{-}=\Omega_{1}-i \Omega_{2}
\end{aligned}
$$

Then (1) be turned into (4), (5), (6)

$$
\begin{aligned}
& \Omega_{3} \Omega^{+}-\Omega^{+} \Omega_{3}=+\Omega^{+} \\
& \Omega_{3} \Omega^{-}-\Omega^{-} \Omega_{3}=-\Omega^{-} \\
& \Omega^{+} \Omega^{-}-\Omega^{-} \Omega^{+}=2 \Omega_{3}
\end{aligned}
$$

Now a group of new symbols is introduced as

$$
\begin{aligned}
& \Omega^{+,-}=\Omega^{+} \Omega^{-} \\
& \Omega^{-,+}=\Omega^{-} \Omega^{+}
\end{aligned}
$$

Then (6) be expressed as

$$
\Omega^{+,-}-\Omega^{-,+}=2 \Omega_{3}
$$

$\Omega^{+,-}$and $\Omega^{-,+}$are matrix products, which are diagonal matrices. We call them matrix contractions.

The types of math symbols (7), (8), (9) are frequently used in this paper.

Base on (7), (8), the marks of $0 \hbar$ boson, $\hbar / 2$ fermion, $1 \hbar$ boson and $3 \hbar / 2$ boson are given as below:

$$
\begin{gathered}
\Delta \quad 0 \hbar \text { boson } \Omega_{j,+0} \\
\Omega_{+0}^{+,-}=\Omega_{+0}^{+} \Omega_{+0}^{-}, \quad \Omega_{+0}^{-,+}=\Omega_{+0}^{-} \Omega_{+0}^{+} ; \quad \Omega_{3,+0}, \Omega_{+0}^{2} \\
\boldsymbol{\nabla} \hbar / 2 \text { fermion } \Omega_{j,+1 / 2} \\
\Omega_{+1 / 2}^{+,-}=\Omega_{+1 / 2}^{+} \Omega_{+1 / 2}^{-}, \quad \Omega_{+1 / 2}^{-,+}=\Omega_{+1 / 2}^{-} \Omega_{+1 / 2}^{+} ; \quad \Omega_{3,+1 / 2}, \Omega_{+1 / 2}^{2} \\
\boldsymbol{A} 1 \hbar \text { boson } \Omega_{j,+1} \\
\Omega_{+1}^{+,-}=\Omega_{+1}^{+} \Omega_{+1}^{-}, \quad \Omega_{+1}^{-,+}=\Omega_{+1}^{-} \Omega_{+1}^{+} ; \quad \Omega_{3,+1}, \Omega_{+1}^{2} \\
\Omega_{+3 / 2}^{+,-}=\Omega_{+3 / 2}^{+} \Omega_{+3 / 2}^{-}, \quad \Omega_{+3 / 2}^{-,+}=\Omega_{+3 / 2}^{-} \Omega_{+3 / 2}^{+} ; \quad \Omega_{3,+3 / 2}, \Omega_{+3 / 2}^{2}
\end{gathered}
$$

The following 2.1, 2.2, 2.3, 2.4 are what we have learned from TKP cite: [2]

And then from paragraphy 3., we are going to explore the origins of bosons $\Omega_{j,+0} \mathbf{\Lambda}, \Omega_{j,+1} \wedge$ and fermions $\Omega_{j,+1 / 2} \mathbf{\nabla}, \Omega_{j,+3 / 2}$. 
2.1. For Boson $0 \hbar$, we have

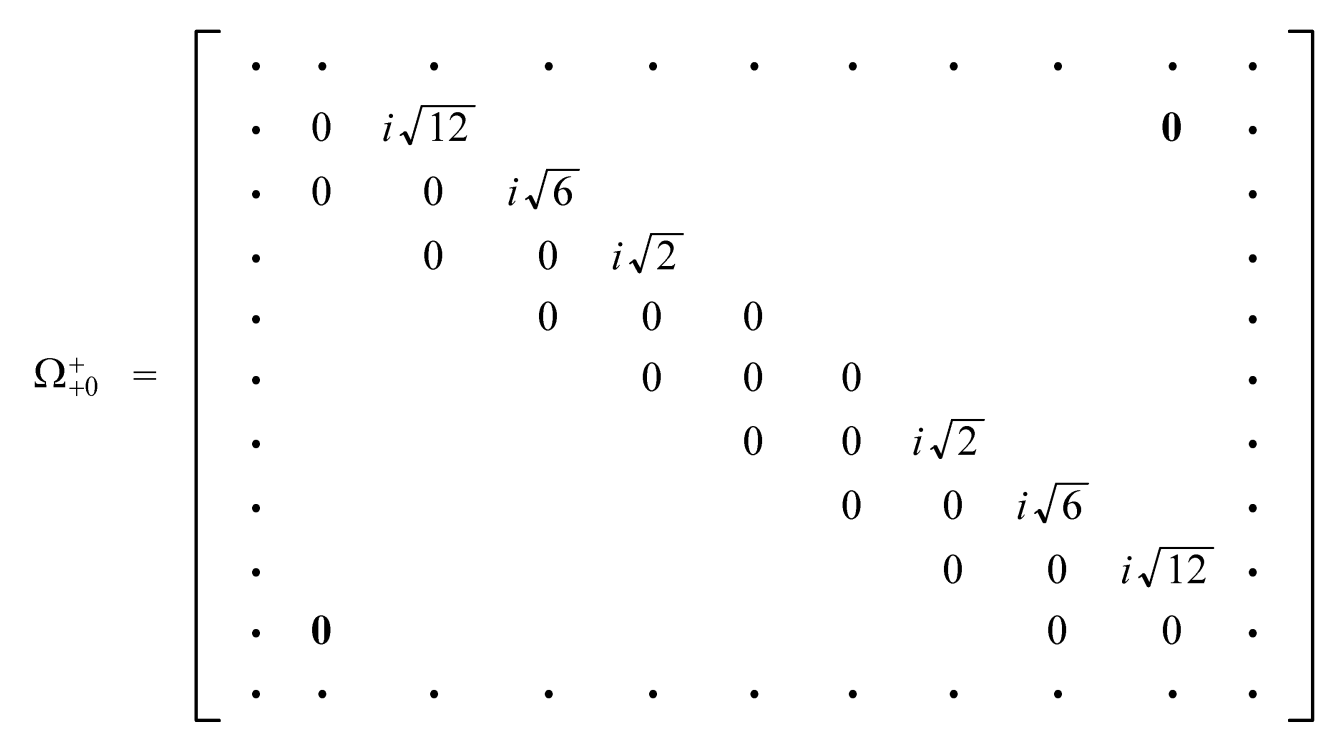

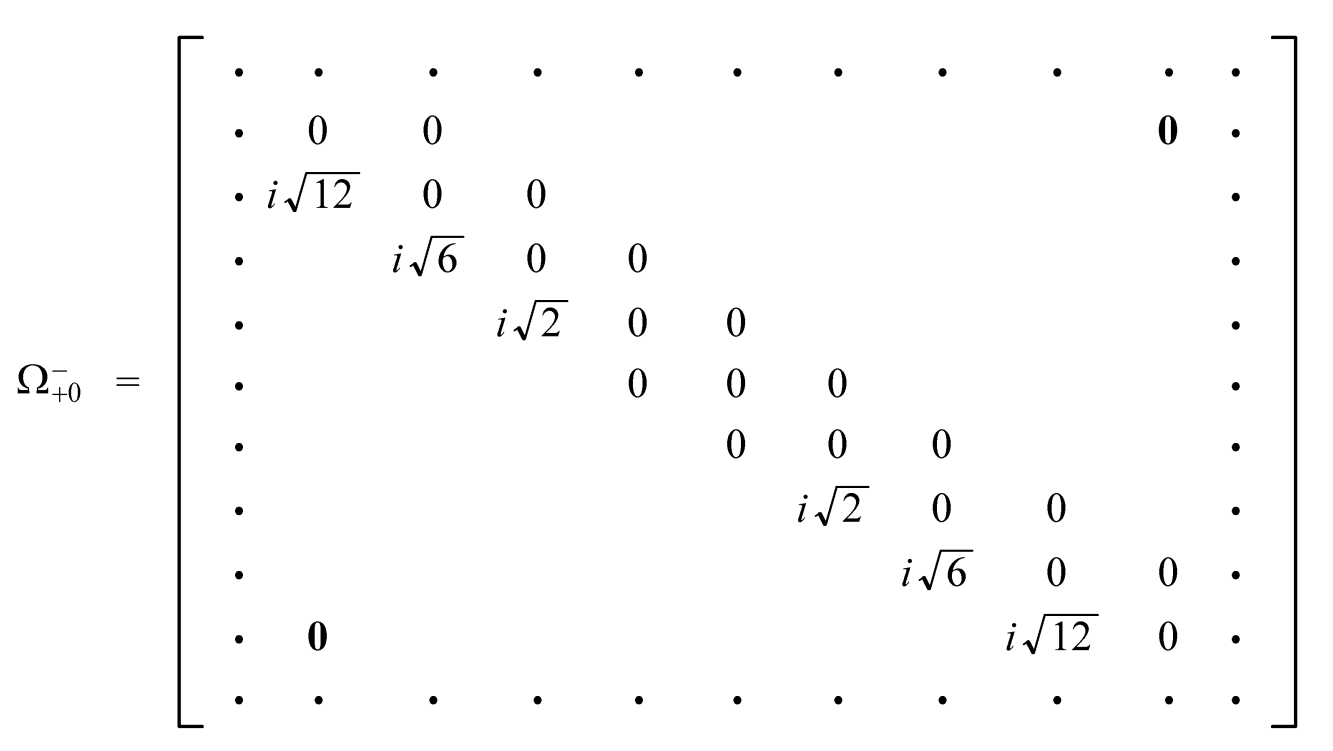

And four diagonal matrices

$$
\begin{array}{lllllllllll}
\Omega_{+0}^{+,-}=\Omega_{+0}^{+} \Omega_{+0}^{-}= & -\operatorname{diag}(\ldots, 12, & 6, & 2, & 0, & 0, & 2, & 6, & 12, & 20, \ldots) \\
\Omega_{+0}^{-++}=\Omega_{+0}^{-} \Omega_{+0}^{+}= & -\operatorname{diag}(\ldots, 20, & 12, & 6, & 2, & 0, & 0, & 2, & 6, & 12, \ldots) \\
\Omega_{3,+0}= & \quad+\operatorname{diag}(\ldots, 4, & 3, & 2, & 1, & 0, & -1, & -2, & -3, & -4, \ldots) \\
\Omega_{+0}^{2}= & \quad+\operatorname{diag}(\ldots, 0, & 0, & 0, & 0, & 0, & 0, & 0, & 0, & 0, \ldots)
\end{array}
$$


2.2. For Boson $\hbar / 2$, we have

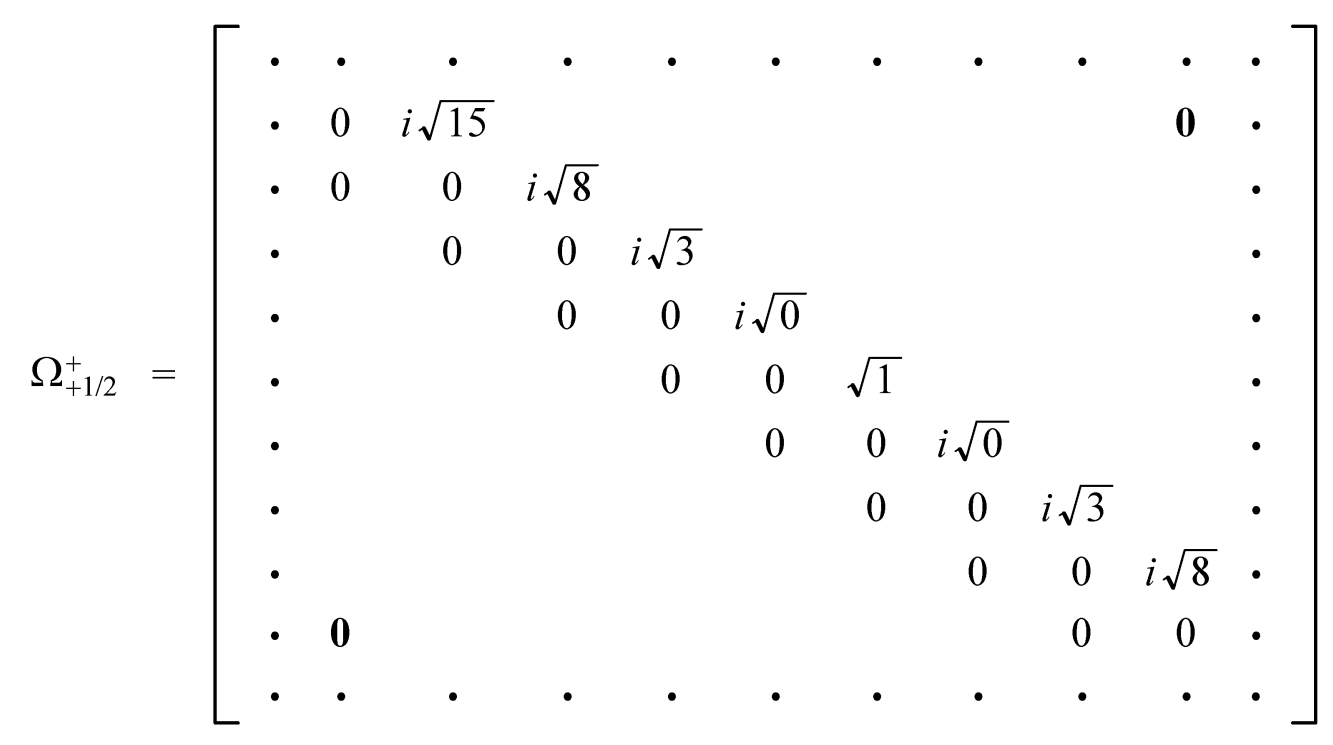

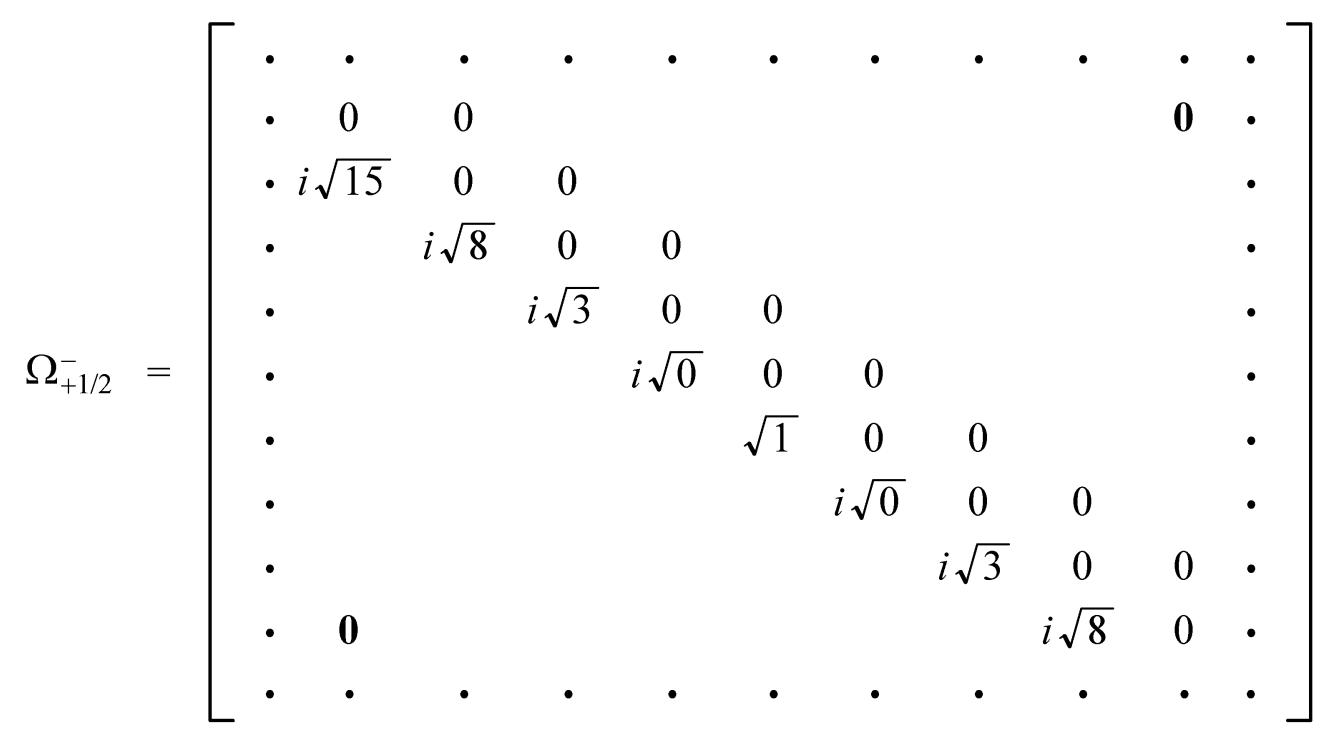

And four diagonal matrices

$$
\begin{aligned}
& \Omega_{+1 / 2}^{+,-}=\Omega_{+1 / 2}^{+} \Omega_{+1 / 2}^{-}=-\operatorname{diag}(\ldots, 15, \quad 8, \quad 3, \quad 0, \quad-1, \quad 0, \quad 3, \quad 8, \quad 15, \ldots) \\
& \Omega_{+1 / 2}^{-,-}=\Omega_{+1 / 2}^{-} \Omega_{+1 / 2}^{+}=-\operatorname{diag}(\ldots, 24,15,8, \quad 3, \quad 0, \quad-1, \quad 0, \quad 3, \quad 8, \ldots) \\
& \Omega_{3,+1 / 2}=\quad+\operatorname{diag}\left(\ldots, \frac{9}{2}, \frac{7}{2}, \frac{5}{2}, \frac{3}{2}, \frac{1}{2},-\frac{1}{2},-\frac{3}{2},-\frac{5}{2},-\frac{7}{2}, \ldots\right) \\
& \Omega_{+1 / 2}^{2}=\quad+\operatorname{diag}\left(\ldots, \frac{3}{4}, \frac{3}{4}, \frac{3}{4}, \frac{3}{4}, \frac{3}{4}, \frac{3}{4}, \frac{3}{4}, \frac{3}{4}, \frac{3}{4}, \ldots\right)
\end{aligned}
$$


2.3. For Boson $1 h$, we have

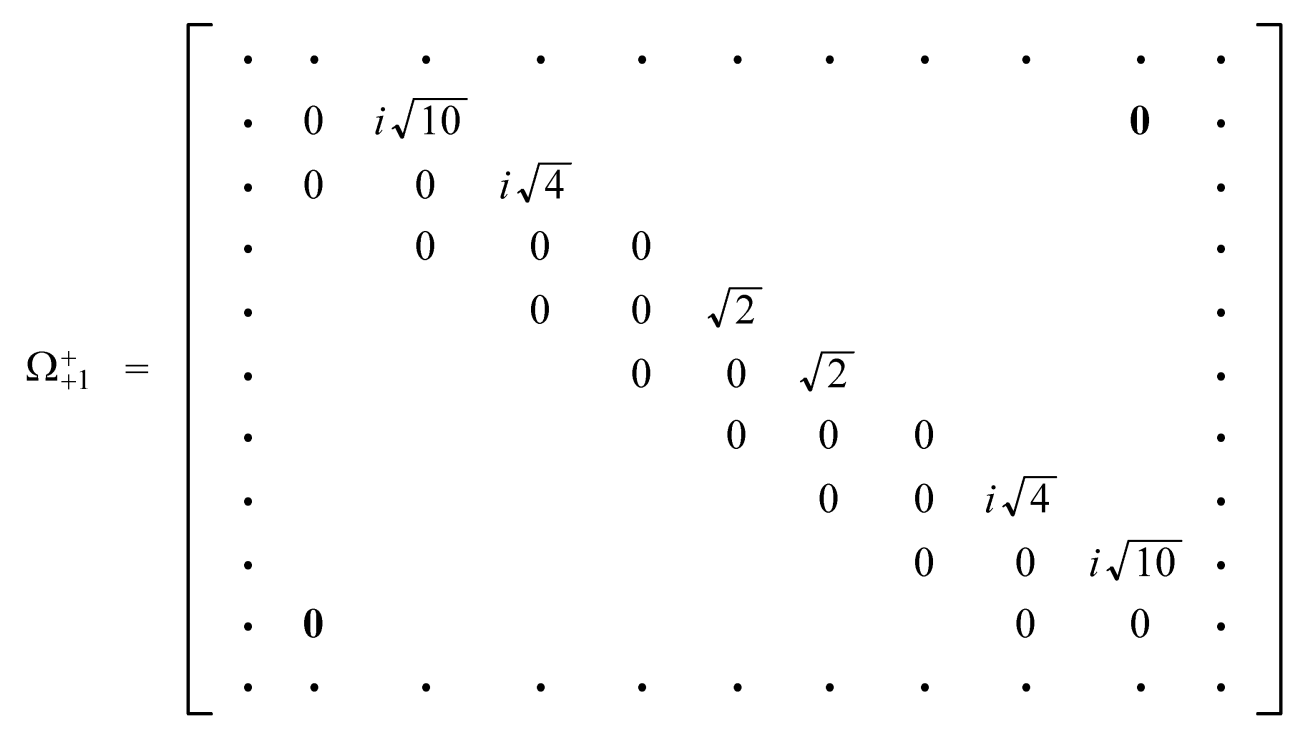

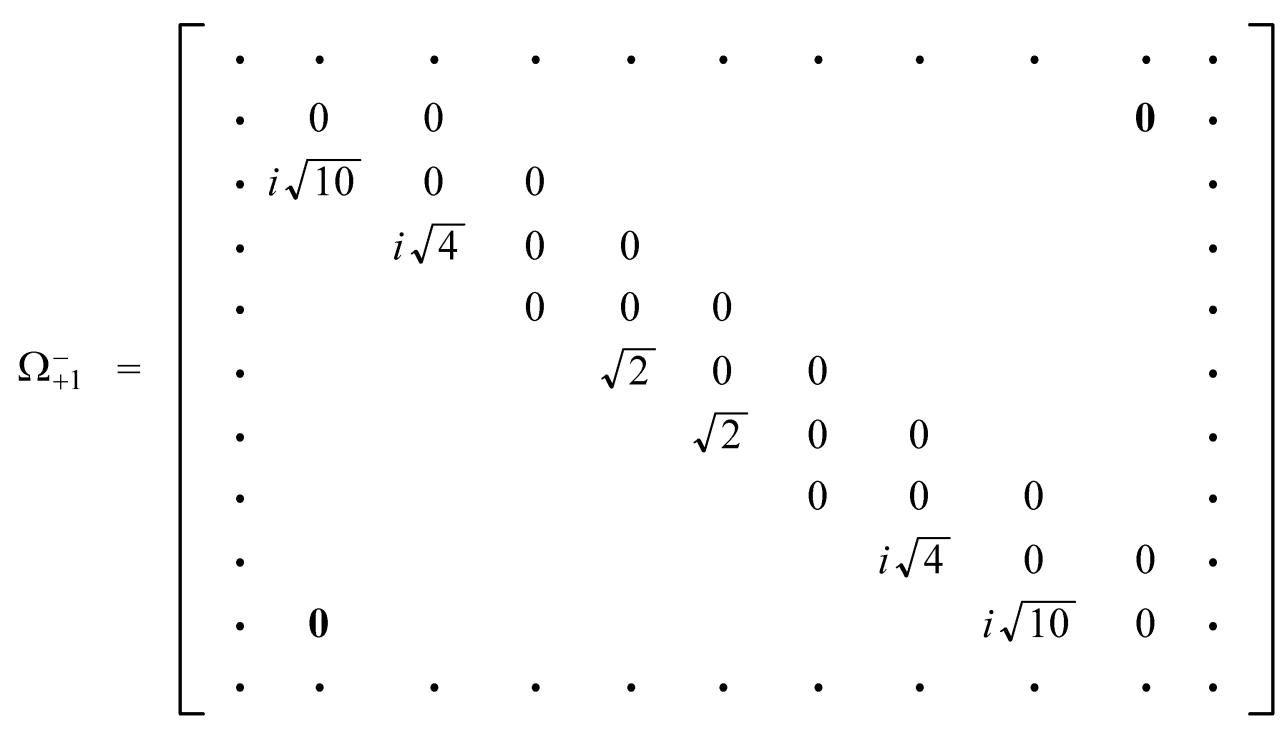

And four diagonal matrices

$$
\begin{aligned}
& \Omega_{+1}^{+,-}=\Omega_{+1}^{+} \Omega_{+1}^{-}=-\operatorname{diag}(\ldots, 10,4,0,-2,-2,0,4,10,18, \ldots) \\
& \Omega_{+1}^{-,-}=\Omega_{+1}^{-} \Omega_{+1}^{+}=-\operatorname{diag}(\ldots, 18,10,4,0,-2,-2,0,4,10, \ldots) \\
& \Omega_{3,+1}=\quad+\operatorname{diag}(\ldots, 4,3,2,1,0,-1,-2,-3,-4, \ldots) \\
& \Omega_{+1}^{2}=\quad+\operatorname{diag}(\ldots, 2,2,2,2,2,2,2,2,2, \ldots)
\end{aligned}
$$


2.4. For Boson $3 \hbar / 2$, we have

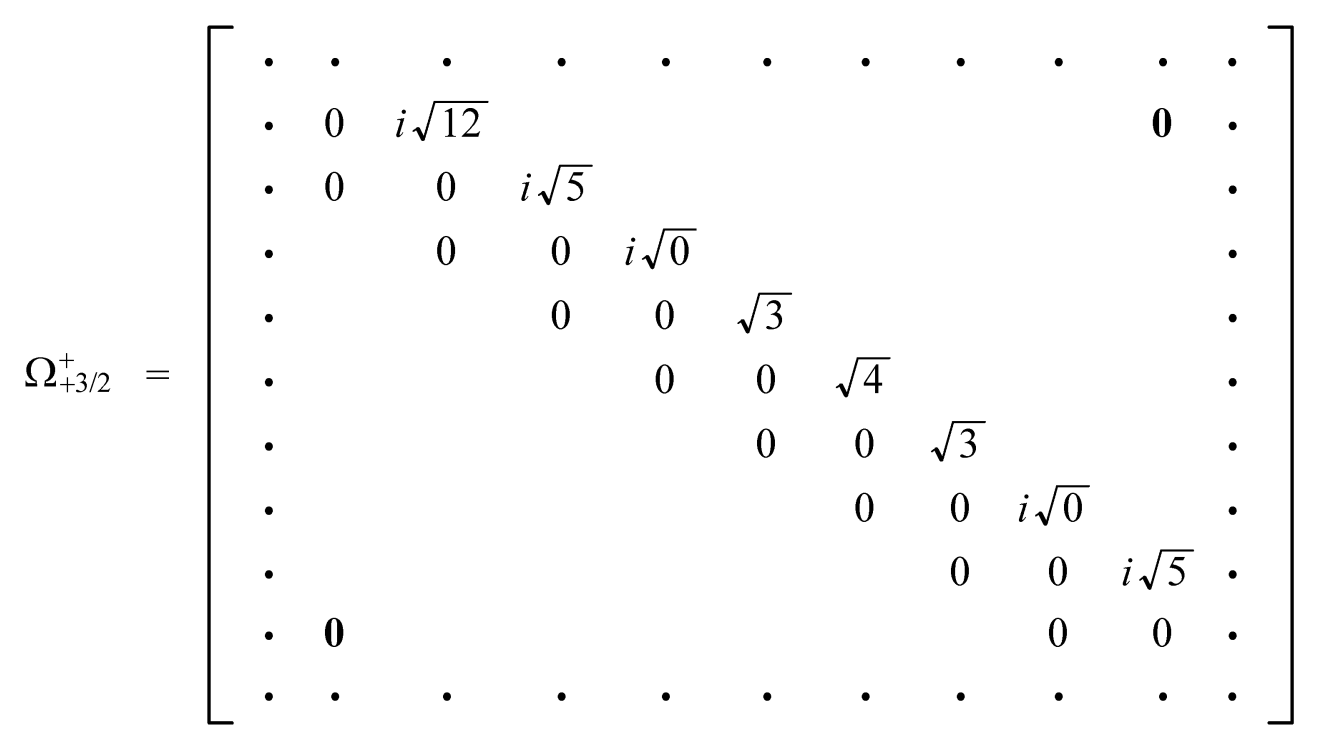

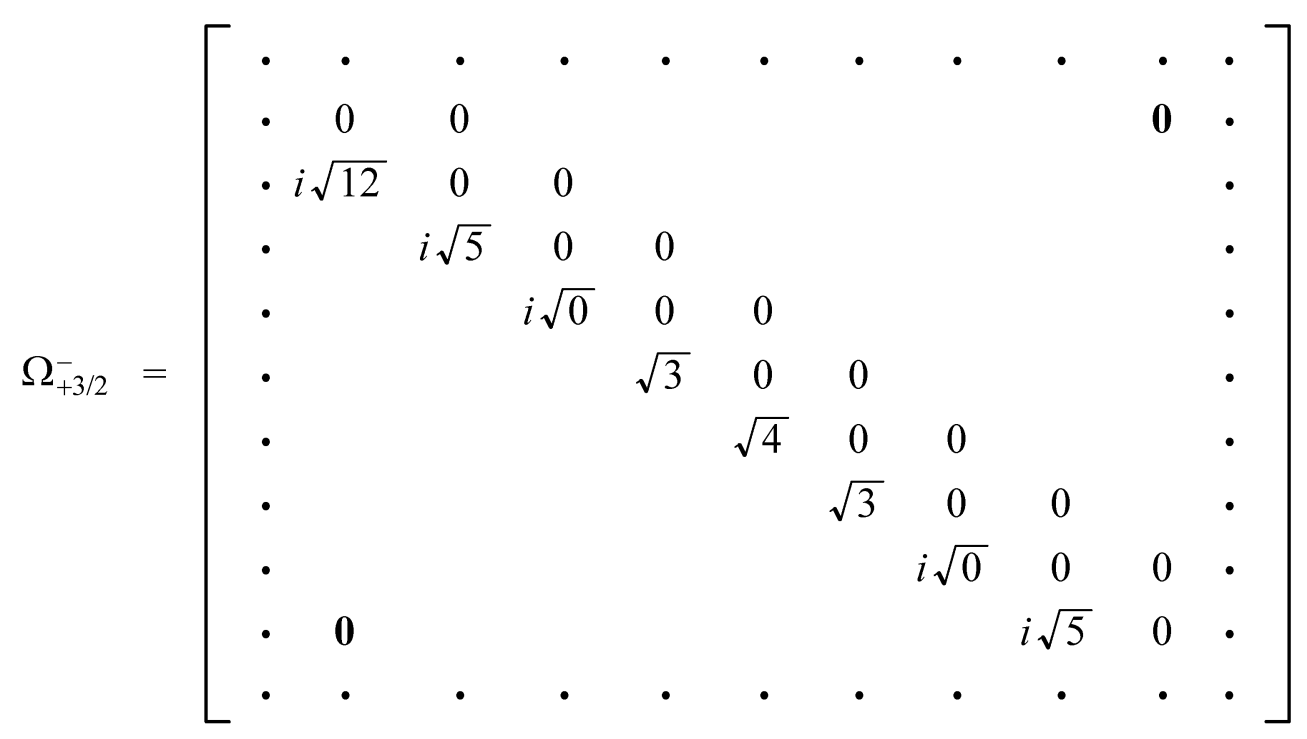

And four diagonal matrices

$$
\begin{aligned}
& \Omega_{+3 / 2}^{+,-}=\Omega_{+3 / 2}^{+} \Omega_{+3 / 2}^{-}=-\operatorname{diag}(\ldots, 12, \quad 5, \quad 0, \quad-3, \quad-4, \quad-3, \quad 0, \quad 5, \quad 12, \ldots) \\
& \Omega_{+3 / 2}^{-,-}=\Omega_{+3 / 2}^{-} \Omega_{+3 / 2}^{+}=-\operatorname{diag}(\ldots, 21,12, \quad 5, \quad 0, \quad-3, \quad-4, \quad-3, \quad 0, \quad 5, \ldots) \\
& \Omega_{3,+3 / 2}=\quad+\operatorname{diag}\left(\ldots, \frac{9}{2}, \frac{7}{2}, \frac{5}{2}, \frac{3}{2}, \frac{1}{2},-\frac{1}{2},-\frac{3}{2},-\frac{5}{2},-\frac{7}{2}, \ldots\right) \\
& \Omega_{+3 / 2}^{2}=\quad+\operatorname{diag}\left(\ldots, \frac{15}{4}, \frac{15}{4}, \frac{15}{4}, \frac{15}{4}, \frac{15}{4}, \frac{15}{4}, \frac{15}{4}, \frac{15}{4}, \frac{15}{4}, \ldots\right)
\end{aligned}
$$




\section{The Flow of This Paper}

1. Introduction

2. Bosons and Fermions in TKP

3. Vacuum Spin Particles VSP

4. Vacuum Bubbles VB and Vacuum Contractions VC

5 Phase Transitions PT of Vacuum Bubbles

6. The formations of Bosons and Fermions

7. Vacuum Bubble Pair VBP $\omega_{\mathrm{S}=|i-j|}^{+,-}(k)$ of the $k$ th generations of CSH

8. Formation of bosons, fermions, TKP and VSP of 2 nd generation of CSH

9. Formation of bosons, fermions, TKP and VSP of 3rd generation of CSH

10. The Sth order phase transitions of Vacuum Bubble Pair in SAMV ocean

11. Spin particles with Casimir Operator $\omega_{\mathrm{S}}^{2}$ formed of Vacuum Bubble Pair $\omega_{\mathrm{S}}^{+,-}$

12. Local and Nonlocal Spin Angular Momentum Commutation Rules

\section{Conclusions}

\section{Vacuum Spin Particles VSP}

Spin Angular Momentum Vacuum SAMV consist of Primitive Spin Particles PSP. SAMV is very deep ocean. About SAMV what This paper concerns is in the shallow water region, its Casimir Operator $\omega^{2}$, which labeled by the negative index $-\frac{1}{2}$, is only negative one-fourth, $-\frac{1}{4} \hbar^{2}$ deep.

VSP is the special and the important case of PSP with index $-\frac{1}{2}$.

Vacuum Spin Particles VSP obey angular momentum commutation rules. Symbols $\omega_{1}(38), \omega_{2}(39), \omega_{3}(40), \omega^{2}(47),(48)$ are the representation of VSP. The Casimir Operator $\omega^{2}$ of VSP is "abnormal", because of its negative $-\frac{1}{4}$ value, instead of those, which labeled by positive or zero values seen in conventional quantum mechanics. 
3.1. From CSH cite: [2], let $\omega_{1}, \omega_{2}, \omega_{3}$ be the generators of VSP

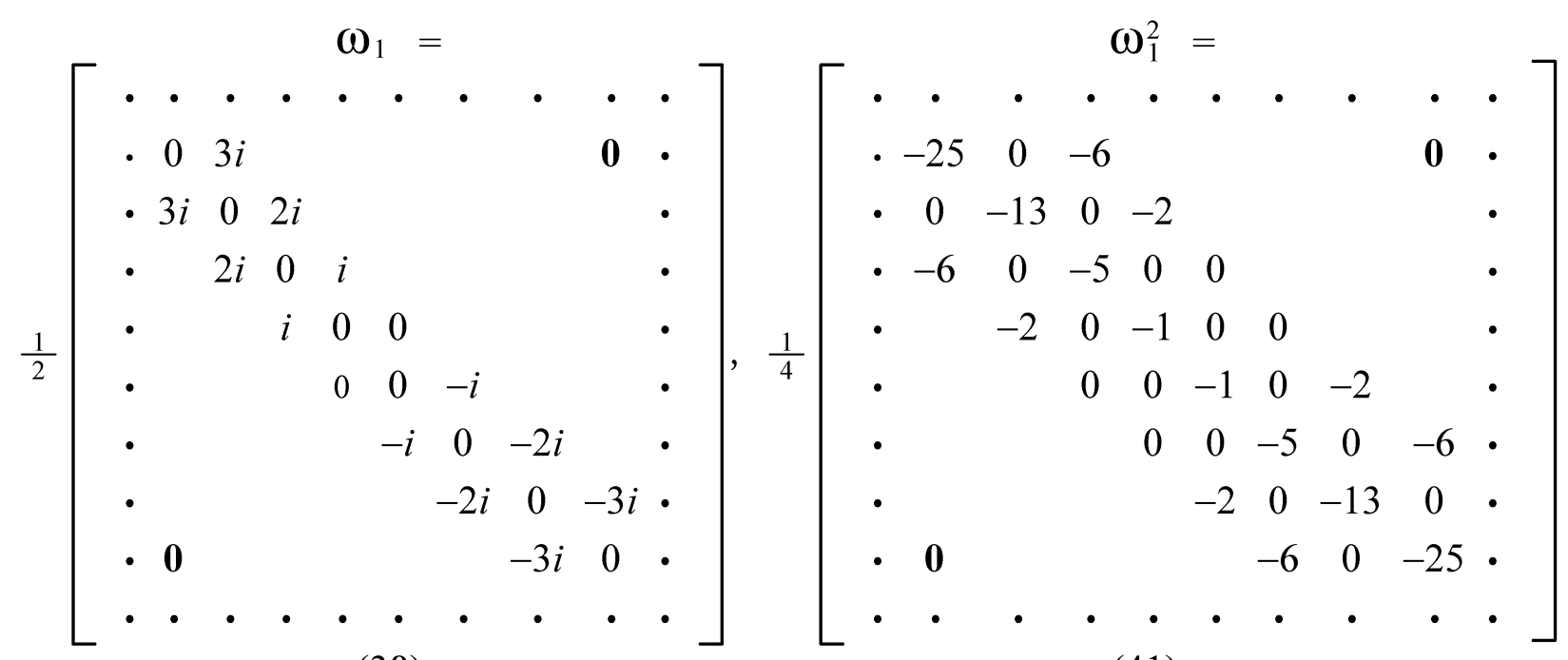

(38)

(41)

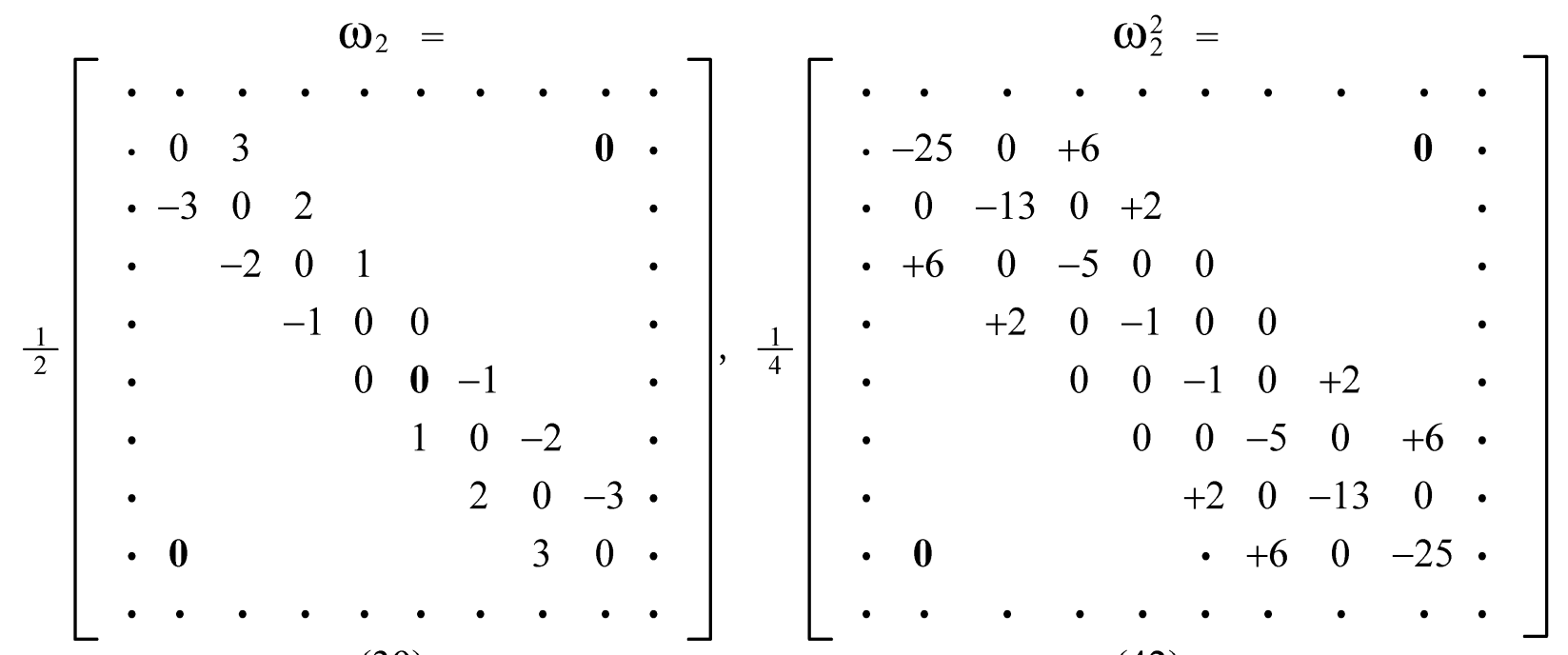

(39)

(42)

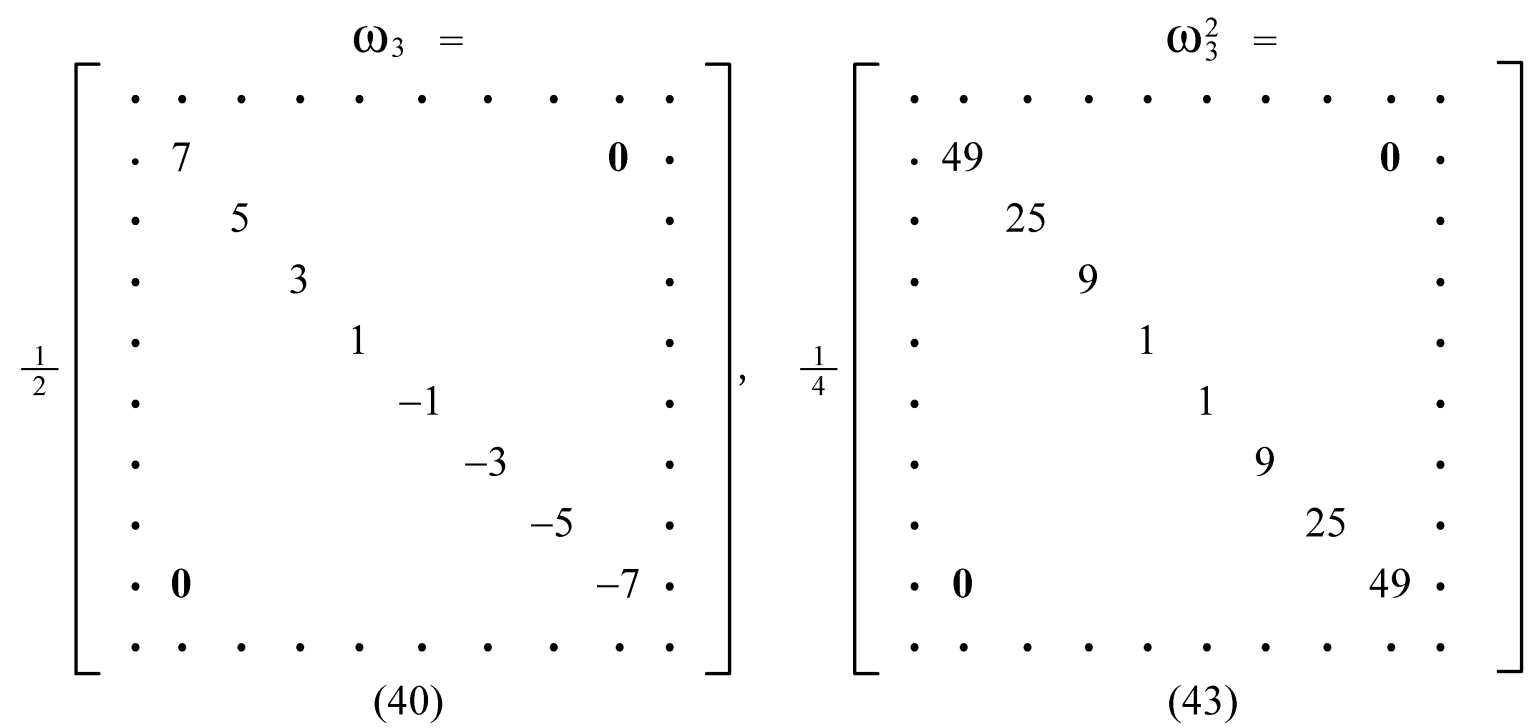


Then we get the following relations:

$$
\begin{array}{cl}
\omega_{1}^{2}+\omega_{2}^{2} & =-\frac{1}{4} \operatorname{diag}(\ldots, 50,26,10,2,2,10,26,50, \ldots) \\
\omega_{3}^{2} & =+\frac{1}{4} \operatorname{diag}(\ldots, 49,25,9,1,1,9,25,49, \ldots) \\
\omega_{1}^{2}+\omega_{2}^{2}+\omega_{3}^{2} & =-\frac{1}{4} \operatorname{diag}(\ldots, 1,1,1,1,1,1,1,1, \ldots)
\end{array}
$$

It is shown: $\omega_{1}(38), \omega_{2}(39)$ are infinite dimensional anti-Hermitian matrices, which lead the sum of $\omega_{1}^{2}+\omega_{2}^{2}$ to beoome a negative diagonal matrix (44) .

And $\omega_{3}(40)$ is an infinite dimensional Hermitian matrix, but absolute value of its square $\omega_{3}^{2}(45)$ is lese than that of $\omega_{1}^{2}+\omega_{2}^{2}$ (44).

Further, the Casimir operator $\omega^{2}$ of (38),(39),(40) VSP, is labeled by the negative index, $-\frac{1}{2}$ below

$$
\begin{gathered}
\omega^{2}=\omega_{1}^{2}+\omega_{2}^{2}+\omega_{3}^{2}=-\frac{1}{4} I_{0}=-\frac{1}{2}\left(-\frac{1}{2}+1\right) I_{0} \\
\omega^{2}=-\frac{1}{4}<0
\end{gathered}
$$

(47) shows: the label of irreducible representation of the Lie group is neither positive nor zero, that contrary to what we often meet in group theory of conventional quantum mechanics.

3.2. Of course, the detailed process of proving the angular momentun commutation rules of (38), (39), (40) VSP shoud be given, befor we use VSP to construct Vacuum Bubbles VB, rest assured!

From (38),(39), have

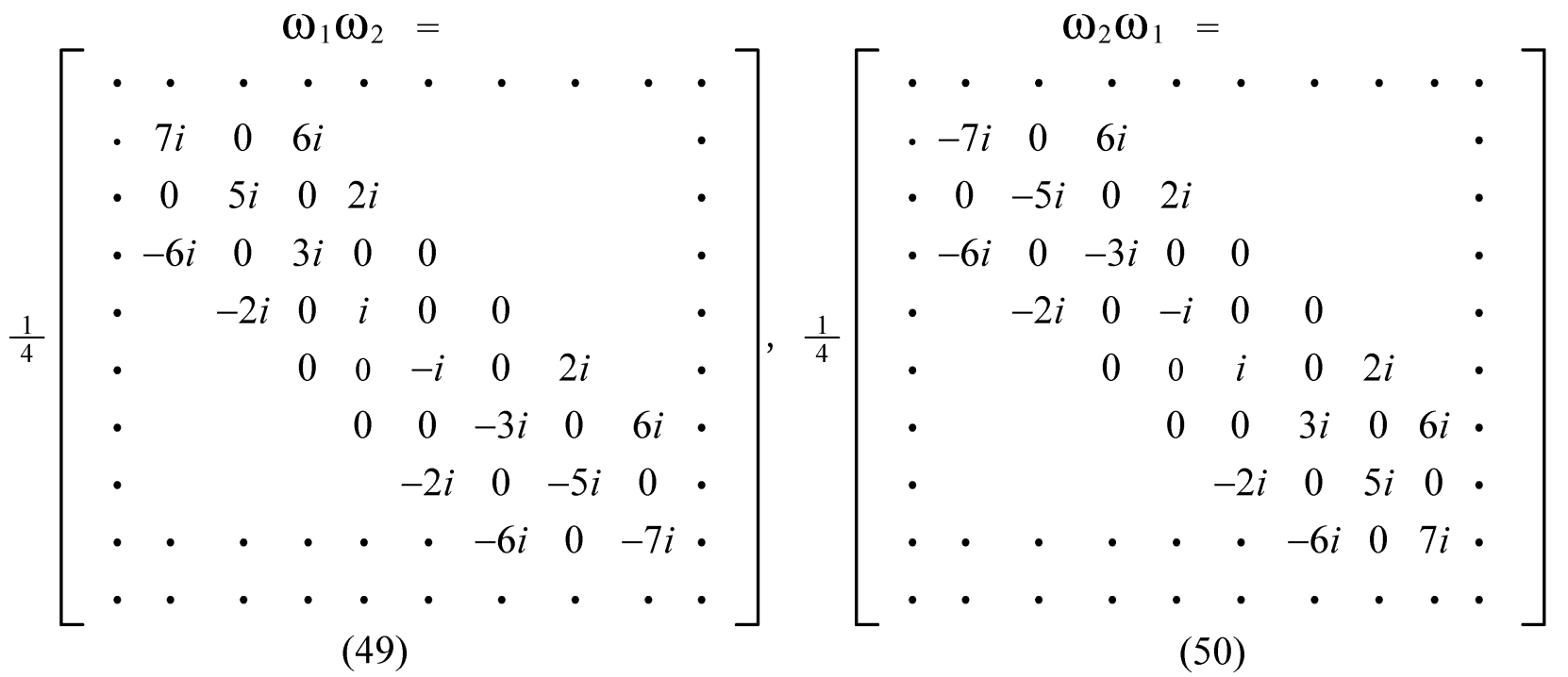


From (39),(40), have

$$
\begin{aligned}
& \omega_{2} \omega_{3}=\quad \omega_{3} \omega_{2}=
\end{aligned}
$$

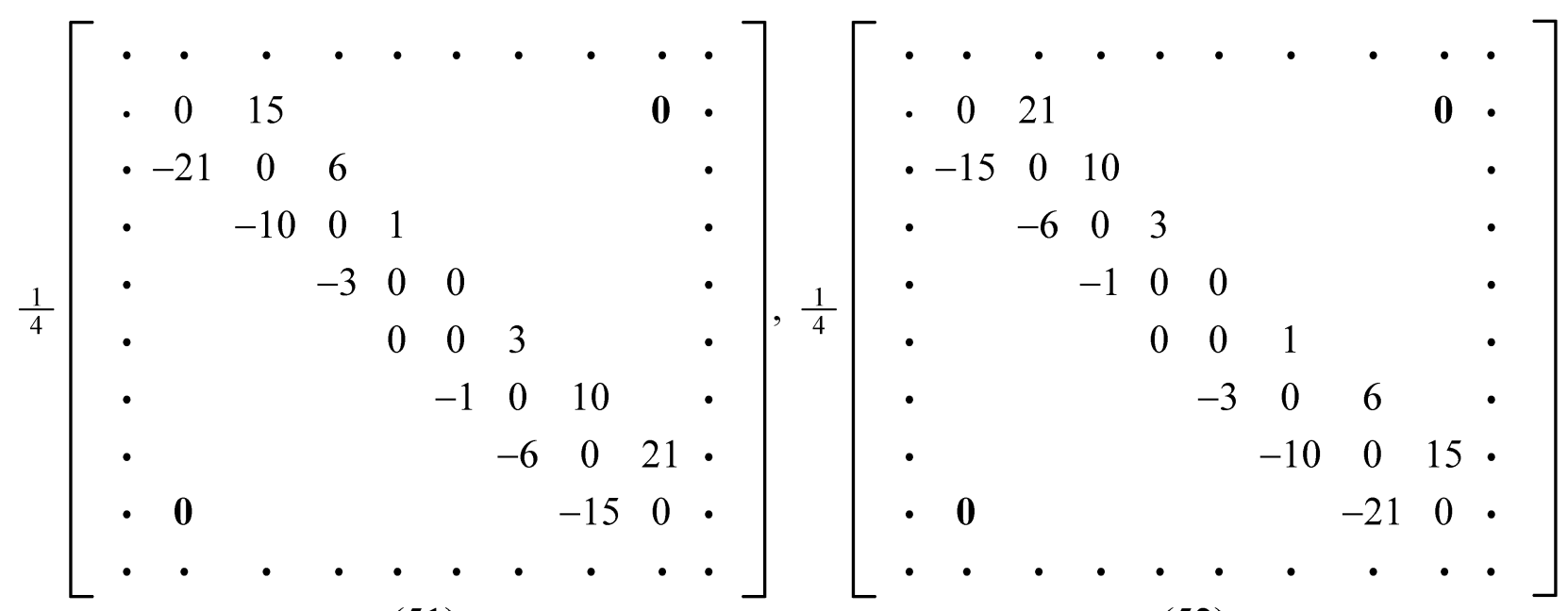

Then obtain $\omega_{2} \omega_{3}+\omega_{3} \omega_{2}$

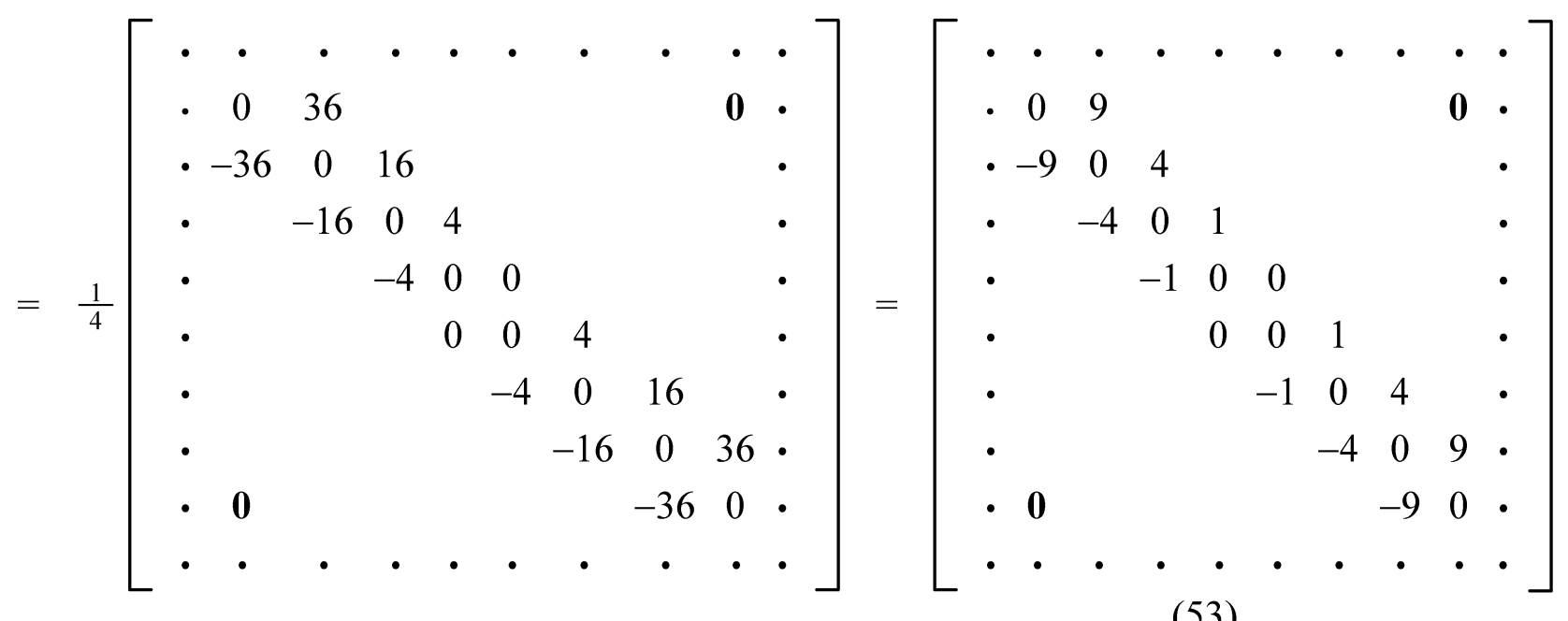

And $\quad \omega_{2} \omega_{3}-\omega_{3} \omega_{2}$

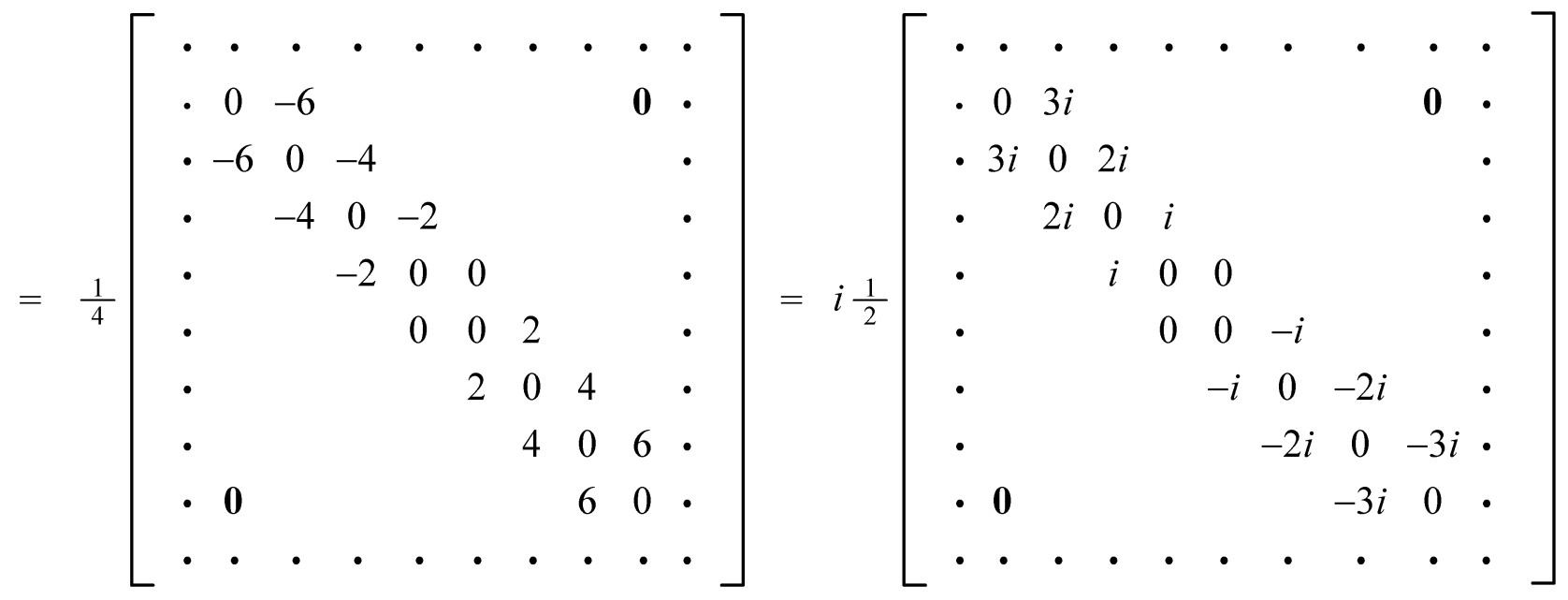$$
=i \omega_{1}
$$ 
From (40),(38), have

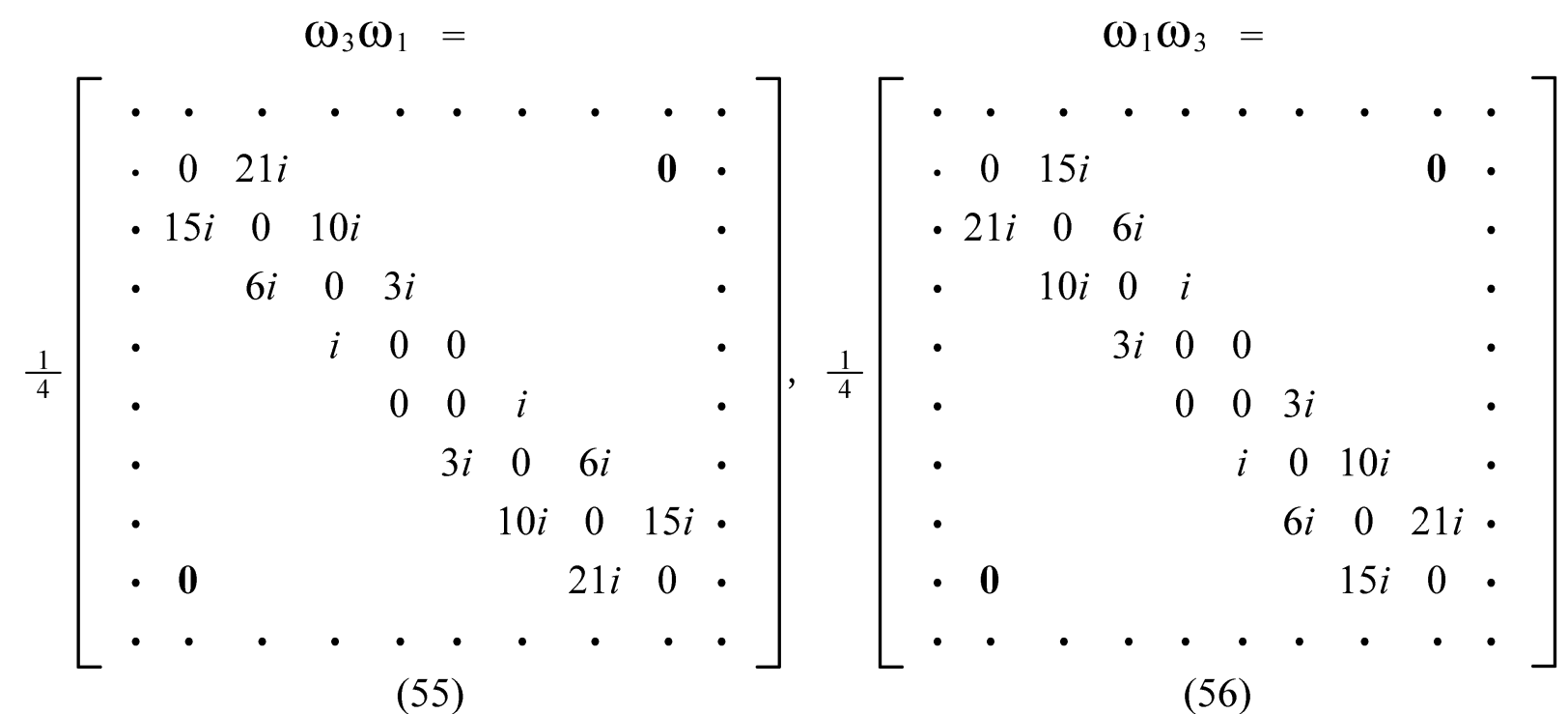

(56)

Then obtain $\omega_{3} \omega_{1}+\omega_{1} \omega_{3}$

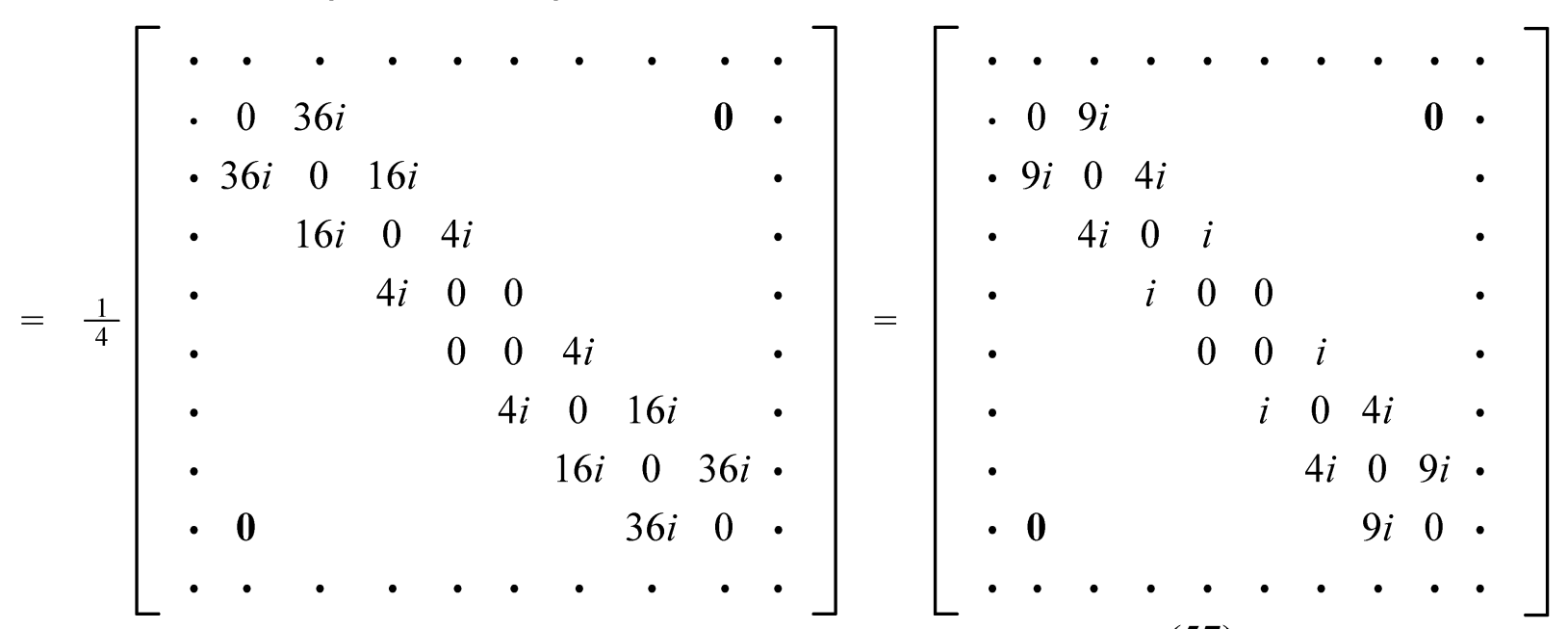

(57)

And $\quad \omega_{3} \omega_{1}-\omega_{1} \omega_{3}$

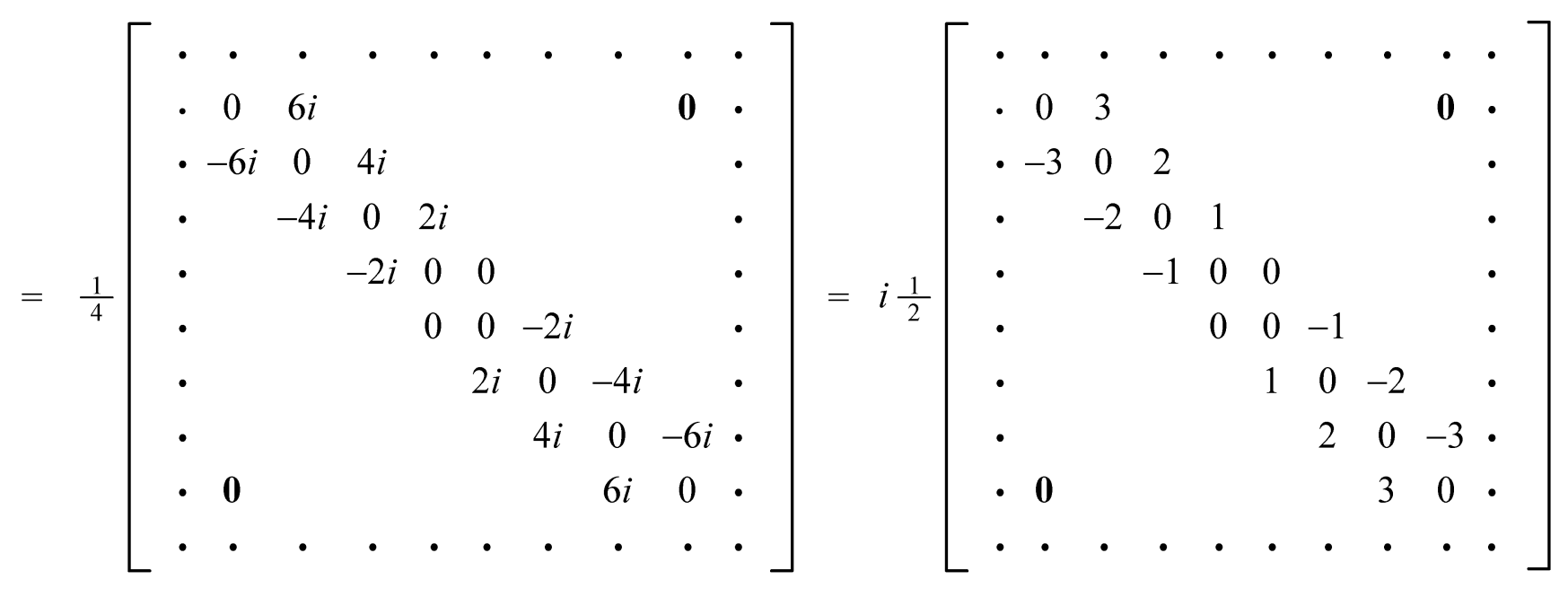

$=i \omega_{2}$

(58) 
From (49), (50) get

$$
\omega_{1} \omega_{2}-\omega_{2} \omega_{1}=\frac{1}{2} i \operatorname{diag}(\ldots, 7,5,3,1,-1,-3,-5,-7, \ldots)=i \omega_{3}
$$

Obviously (59), (54), (58) show $\omega_{1}, \omega_{2}, \omega_{3}$ satisfy angular momentum commutation rules.

$$
\omega_{j} \omega_{k}-\omega_{k} \omega_{j}=i \omega_{l}, \quad j, k, l=1,2,3
$$

Further, $\omega_{1}, \omega_{2}, \omega_{3}$ are angular momentum operators,

From (49), (50) get

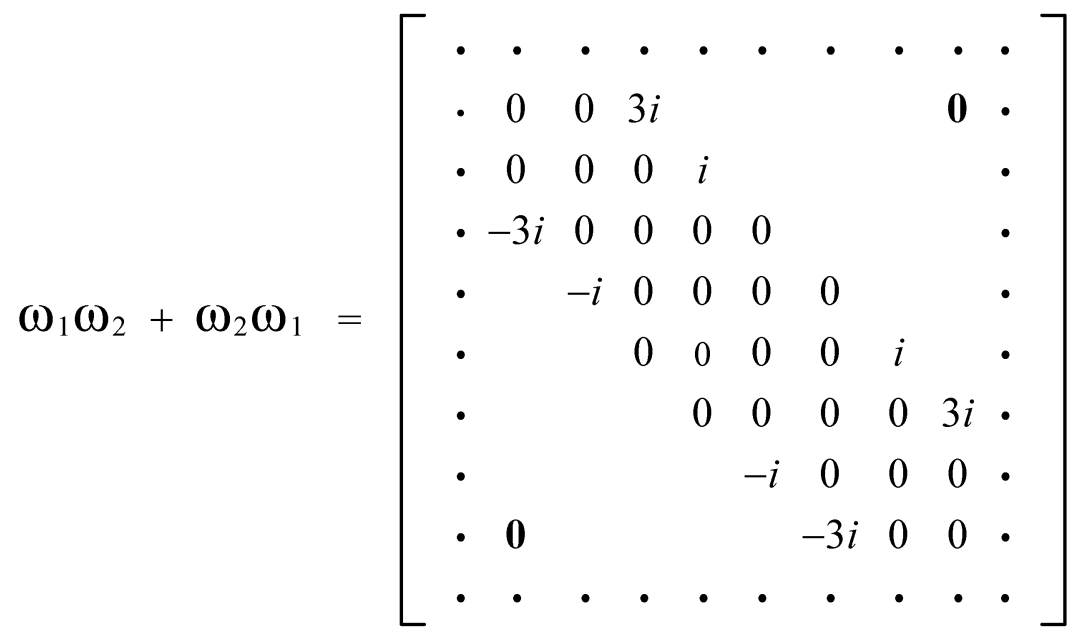

a) As the label $-\frac{1}{2}$ (47) of irreducible representation, further $\omega_{1}, \omega_{2}, \omega_{3}$ are named $-\frac{1}{2} \hbar$ spin particle.

b) As $-\frac{1}{4}<0$ (46) of Casimir operator $\omega^{2}$, and momentum commutation rules (23), further (38), (39), (40) are called Vacuum Spin Particles, VSP.

Recall conventional Fermion, example of electron, its label of irreducible representation is $+\frac{1}{2}$ below

$$
\begin{gathered}
\omega_{\text {electron }}^{2}=+\frac{3}{4} I_{0}=+\frac{1}{2}\left(+\frac{1}{2}+1\right) I_{0} \\
\omega^{2}=+\frac{3}{4}>0
\end{gathered}
$$

c) As the label $+\frac{1}{2}$ (62) of irreducible representation, further electron is named $+\frac{1}{2} \hbar$ spin particle.

By the way, (61) is different from (53) and (57), the former is an infinite Hermitian matrix and the latter two are infinite anti-Hermitian matrices. 


\section{Vacuum Bubbles VB and Vacuum Contractions VC}

$$
\omega^{+,-}, \omega^{-,+} \text {are Vacuum Bubbles and Vacuum Contractions }
$$

Let us perform the following change of bases (38), (39) of VSP

$$
\begin{aligned}
& \omega^{+}=\omega_{1}+i \omega_{2} \\
& \omega^{-}=\omega_{1}-i \omega_{2}
\end{aligned}
$$

Then get raising operator $\omega^{+}$and lowing operator $\omega^{-}$.

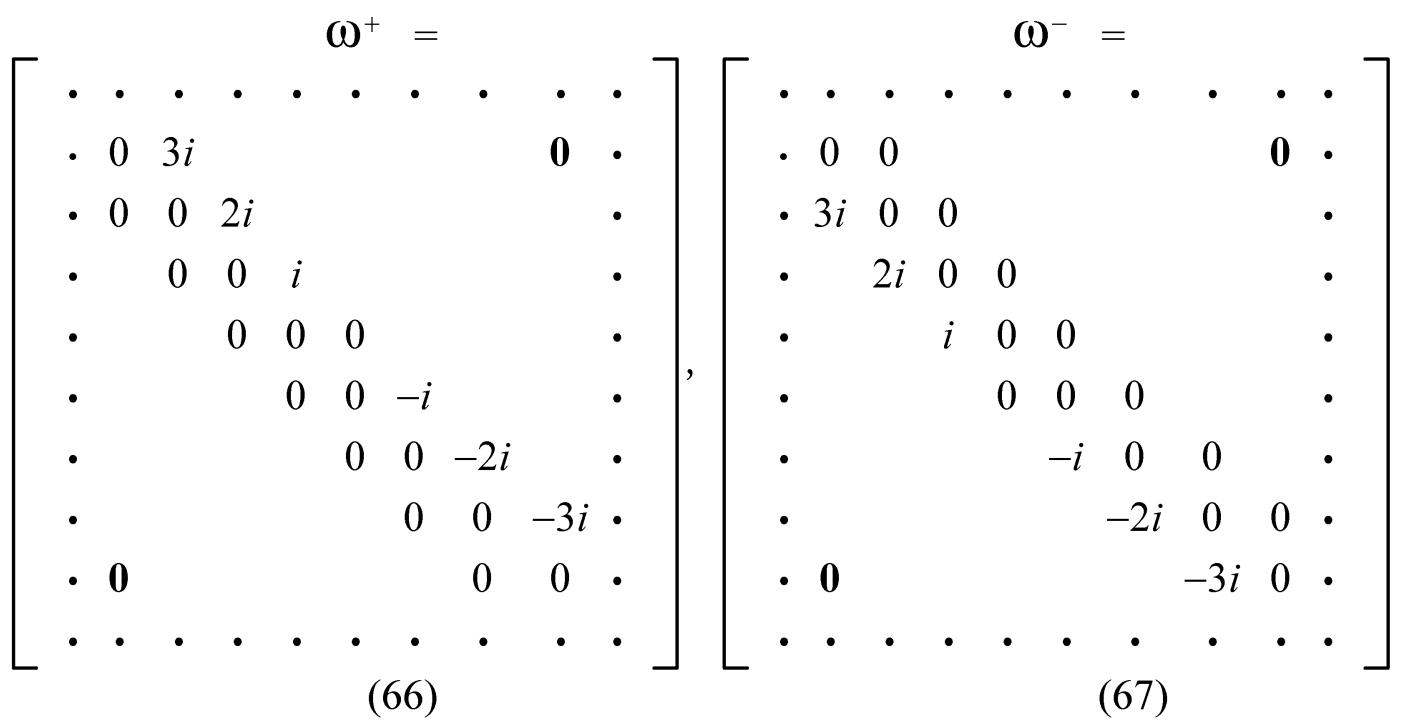

And have the following useful relations:

$$
\begin{aligned}
\omega^{+} \omega^{-} & =\omega_{1}^{2}+\omega_{2}^{2}+\omega_{3} \\
\omega^{-} \omega^{+} & =\omega_{1}^{2}+\omega_{2}^{2}-\omega_{3} \\
\omega_{1}^{2}+\omega_{2}^{2} & =\frac{1}{2}\left(\omega^{+} \omega^{-}+\omega^{-} \omega^{+}\right) \\
\omega_{3} & =\frac{1}{2}\left(\omega^{+} \omega^{-}-\omega^{-} \omega^{+}\right) \\
\omega^{2} & =\omega_{1}^{2}+\omega_{2}^{2}+\omega_{3}^{2}
\end{aligned}
$$

From (66), (67) obtain

$$
\begin{aligned}
& \omega^{+} \omega^{-}=-\operatorname{diag}(\ldots, 9,4,1,0,1,4,9,16, \ldots) \\
& \omega^{-} \omega^{+}=-\operatorname{diag}(\ldots, 16,9,4,1,0,1,4,9, \ldots)
\end{aligned}
$$


Using (73), (74), from (71), obtain

$$
\begin{aligned}
\omega_{3} & =\frac{1}{2} \operatorname{diag}(\ldots, 7,5,3,1,-1,-3,-5,-7, \ldots) \\
& =\operatorname{diag}\left(\ldots, \frac{7}{2}, \frac{5}{2}, \frac{3}{2}, \frac{1}{2},-\frac{1}{2},-\frac{3}{2},-\frac{5}{2},-\frac{7}{2}, \ldots\right) \\
\omega_{3}^{2} & =+\operatorname{diag}\left(\ldots, \frac{49}{4}, \frac{25}{4}, \frac{9}{4}, \frac{1}{4}, \frac{1}{4}, \frac{9}{4}, \frac{25}{4}, \frac{49}{4}, \ldots\right)
\end{aligned}
$$

Using (73), (74), from (70), obtain

$$
\begin{aligned}
\omega_{1}^{2}+\omega_{2}^{2} & =-\frac{1}{2} \operatorname{diag}(\ldots, 25,13,5,1,1,5,13,25, \ldots) \\
& =-\operatorname{diag}\left(\ldots, \frac{50}{4}, \frac{26}{4}, \frac{10}{4}, \frac{2}{4}, \frac{2}{4}, \frac{10}{4}, \frac{26}{4}, \frac{50}{4}, \ldots\right)
\end{aligned}
$$

Then, (72) and (76), (77) give the Casimir Operator $\omega^{2}$ labeled by the negative index $-\frac{1}{2}$

$$
\begin{aligned}
\omega^{2} & =\operatorname{diag}\left(\ldots,-\frac{1}{4},-\frac{1}{4},-\frac{1}{4},-\frac{1}{4},-\frac{1}{4},-\frac{1}{4},-\frac{1}{4},-\frac{1}{4}, \ldots\right) \\
& =-\frac{1}{4} I_{0}=-\frac{1}{2}\left(-\frac{1}{2}+1\right) I_{0}
\end{aligned}
$$

Expressions (75), (79) are just (40), (47).

$\omega^{+} \omega^{-}(73), \omega^{-} \omega^{+}$(74) are in the form of diagonal of matrix products, whose values are negative. What are the roles of (73), (74) in SAMV ocean ?

\subsection{Vacuum Bubbles VB and Vacuum Contractions VC of VSP}

The above process hints the matrix products $\omega^{+} \omega^{-}(73), \omega^{-} \omega^{+}$(74) are the marvellous concepts connected with diagonal matrices.

We call them matrix contractions, or Vacuum Contractions VC or Vacuum Bubbles VB.

Now use compact notions (80), (81) to define Vacuum Contraction of a drop of Vacuum Bubbles in SAMV ocean.

$$
\begin{aligned}
& \text { Vacuum Contraction } \omega^{+,-} \equiv \text { Matrix Product } \omega^{+} \omega^{-} \\
& \text {Vacuum Contraction } \omega^{-,+} \equiv \text { Matrix Product } \omega^{-} \omega^{+}
\end{aligned}
$$

In SAMV ocean, there are many, many... drops of Vacuum Bubbles which resulted from the contracted Vacuum Spin Particles VSP.

These bubbles are the individual bubbles, are so-called "identical bubbles" juat as "identical particles" in statistical physics world.

Symbol $j$ is introduced to indicate the amounts of many, many...individual bubbles. 


\subsection{Self-identical bubble Vacuum contractions}

We use single subscript $j$ to express Vacuum Spin Particles $\omega_{j}$. And double subscripts $j, j$ to label the identical bubbles $\omega_{j, j}^{+,-}(84)$ and $\omega_{j, j}^{-,+}(85)$ composed of Vacuum Spin Particle $\omega_{j}$

$$
\begin{aligned}
& \omega_{j}^{+}=\omega_{j, 1}+i \omega_{j, 2} \\
& \omega_{j}^{-}=\omega_{j, 1}-i \omega_{j, 2}
\end{aligned}
$$

Where $\omega_{j, 1}$ and $\omega_{j, 2}$ are the first component and the second component of Vacuum Spin Particle $\omega_{j}$.

Further, contractions (80), (81) can be extended to the following expressions

$$
\begin{array}{ll}
\text { Vacuum Contraction of identical bubble } j & \omega_{j, j}^{+,-} \equiv \omega_{j}^{+} \omega_{j}^{-} \\
\text {Vacuum Contraction of identical bubble } j & \omega_{j, j}^{-,+} \equiv \omega_{j}^{-} \omega_{j}^{+}
\end{array}
$$

The appearance of subscript $j$ in (84), (85) will bring many magical and profound ideas to modern physics.

Vacuum contractions (84), (85) also be called Self-identical bubble Vacuum contractions, because the two subscripts appear in $\omega_{j, j}^{+,-}(84)$ and $\omega_{j, j}^{-,+}(85)$ are the same symbol $j$.

Due to symbol $j$ of (84) and (85) is referred to any drop of Vacuum Bubbles in SAMV ocean, obviously, symbol $j$ can be all the positive number, zero, negative number. From now on, symbol $j=0$ is chosen to represent the indicate Self-identical bubble Vacuum contractions we are caring about.

As contractions (73), (74) are attributed to Self-identical bubble Vacuum contractions, further they can be rewritten as below

$$
\begin{aligned}
& \omega_{0,0}^{+,-}=\omega_{0}^{+} \omega_{0}^{-}=-\operatorname{diag}(\ldots, 25,16,9,4,1,0,1,4,9,16,25, \ldots) \\
& \omega_{0,0}^{-,+}=\omega_{0}^{-} \omega_{0}^{+}=-\operatorname{diag}(\ldots, 36,25,16,9,4,1,0,1,4,9,16, \ldots)
\end{aligned}
$$

Using vacuum contractions (86), (87), all the informations of irreducible representation labeled by the negative index $-\frac{1}{2}$, can be obtained. 


\subsection{Dual-identical bubble Vacuum contractions}

How about the bubble Vacuum contractions, if " From the self-action of one drop of Bubbles to the dual-action of all drops of Bubbles " ?

Provide that subscript $i \neq j$, then (84), (85) turn to

$$
\begin{array}{ll}
\text { Contraction between bubble } i \text { and bubble } j & \omega_{i, j}^{+,-} \equiv \omega_{i}^{+} \omega_{j}^{-} \\
\text {Contraction between bubble } j \text { and bubble } i & \omega_{j, i}^{-,+} \equiv \omega_{j}^{-} \omega_{i}^{+}
\end{array}
$$

In process that similar to Self-identical bubble Vacuum contractions (86), (87), then four Dual-identical bubble Vacuum contractions are given in following. More details can be founded from cite: [3]

$$
\begin{gathered}
\omega_{+1,0}^{+,-}=\omega_{+1}^{+} \omega_{0}^{-}=-\operatorname{diag}(\ldots, 20,12,6,2,0,0,2,6,12,20,30, \ldots) \\
\omega_{0,+1}^{-,+}=\omega_{0}^{-} \omega_{+1}^{+}=-\operatorname{diag}(\ldots, 30,20,12,6,2,0,0,2,6,12,20, \ldots) \\
\omega_{+1,-1}^{+,-}=\omega_{+1}^{+} \omega_{-1}^{-}=-\operatorname{diag}(\ldots, 24,15,8,3,0,-1,0,3,8,15,24, \ldots) \\
\omega_{-1,+1}^{-,+}=\omega_{-1}^{-} \omega_{+1}^{+}=-\operatorname{diag}(\ldots, 35,24,15,8,3,0,-1,0,3,8,15, \ldots) \\
\omega_{+2,-1}^{+,-}=\omega_{+2}^{+} \omega_{-1}^{-}=-\operatorname{diag}(\ldots, 18,10,4,0,-2,-2,0,4,10,18,28, \ldots) \\
\omega_{-1,+2}^{-,+}=\omega_{-1}^{-} \omega_{+2}^{+}=-\operatorname{diag}(\ldots, 28,18,10,4,0,-2,-2,0,4,10,18, \ldots) \\
\omega_{+2,-2}^{+,-}=\omega_{+2}^{+} \omega_{-2}^{-}=-\operatorname{diag}(\ldots, 21,12,5,0,-3,-4,-3,0,5,12,21, \ldots) \\
\omega_{-2,+2}^{-,+}=\omega_{-2}^{-} \omega_{+2}^{+}=-\operatorname{diag}(\ldots, 32,21,12,5,0,-3,-4,-3,0,5,12, \ldots)
\end{gathered}
$$

Next, the above four groups of contractions or bubbles will be used to synthesize the known bosons and fermions of today's physics by means of phase transitions of Vacuum Bubbles.

\section{Phase Transitions PT of Vacuum Bubbles}

Referring to Phase transitions of Vacuum Bubbles, it means the processes in which the sub-observable physical quantities, vacuum contractions $\boldsymbol{\omega}_{i, j}^{+,-}$ and $\omega_{j, i}^{-,+}$, are combined into observable physical quantities, $\omega_{1 ; i, j}^{2}+\omega_{2 ; i, j}^{2}$ and $\omega_{3 ; i, j}, \quad \omega_{3 ; i, j}^{2}$, by (70), (71), (72).

In Dual-identical bubble Vacuum contractions, Subscript $i$ and subscript $j$ are satisfied with the following formula (refer. paragraph 6.)

$$
\mathrm{S}=|i-j|=1,2,3,4,5, \ldots \ldots
$$

For Self-identical bubble Vacuum contractions, $\mathrm{s}=0$.

From next paragraph, we begin to explain how bosons and fermions are formed from phase transitions of Dual-identical Bubbles $(S \neq 0)$. 


\section{The formations of Bosons and Fermions}

6.1

Formation of $0 \hbar$ bosons result in

the first order Vacuum Bubble phase transition

by $\omega_{+1,0}^{+,-}$and $\omega_{0,+1}^{-,+}$

From

Table1: Vacuum Contractions (90),(91)

$\omega_{+1,0}^{+,-}=$

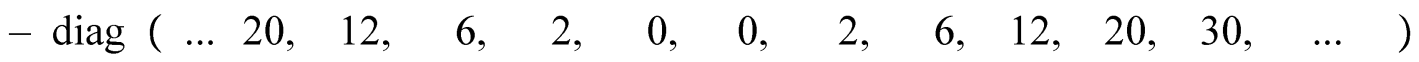

$\omega_{0,+1}^{-,+}=$

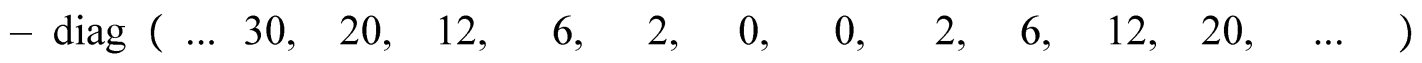

Obtain

Table2: $\omega_{1 ;+1,0}^{2}+\omega_{2 ;+1,0}^{2}$

$\omega_{1 ;+1,0}^{2}+\omega_{2 ;+1,0}^{2}=\frac{1}{2}\left(\omega_{+1,0}^{+,-}+\omega_{0,+1}^{-,+}\right)=$

$-\frac{1}{2} \operatorname{diag}(\ldots \quad 50, \quad 32, \quad 18, \quad 8, \quad 2, \quad 0, \quad 2, \quad 8, \quad 18, \quad 32, \quad 50, \quad \ldots \quad)$

$=$

$\operatorname{diag}(\ldots-25,-16,-9,-4,-1,0,+1,-4,-9,-16,-25, \ldots)$

And

Table3: $\omega_{3 ;+1,0}, \omega_{3 ;+1,0}^{2}$

$\omega_{3 ;+1,0}=\frac{1}{2}\left(\omega_{+1,0}^{+,-}-\omega_{0,+1}^{-,+}\right)=$

$+\frac{1}{2} \operatorname{diag}\left(\begin{array}{lllllllllllll}\ldots & 10, & 8, & 6 & 4, & 2 & 0, & -2, & -4, & -6, & -8, & -10, & \ldots\end{array}\right)$

$=\operatorname{diag}(\ldots+5, \quad+4,+3,+2,+1,0,-1,-2,-3,-4, \quad-5, \quad \ldots)$

$\omega_{3 ;+1,0}^{2}=$

$\operatorname{diag}(\ldots+25,+16,+9,+4,+1,0,+1,+4,+9,+16,+25, \ldots)$

Then

Table4: $\omega_{+1,0}^{2}$

$\omega_{+1,0}^{2}=\omega_{1 ;+1,0}^{2}+\omega_{2 ;+1,0}^{2}+\omega_{3 ;+1,0}^{2}=$

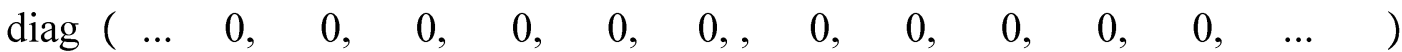

$=0 I_{0}=0(0+1) I_{0}$

Table $3 \omega_{3 ;+1,0}$ and Table4: $\omega_{+1,0}^{2}$ are just the known $0 \hbar$ boson of CSH !

$$
\omega_{+1,0}^{2}=\Omega_{+0}^{2} \quad \omega_{3,+1,0}=\Omega_{3,+0} \quad ! ! !
$$




\section{2}

Formation of $\hbar / 2$ fermions result in the second order Vacuum Bubble phase transition by $\omega_{+1,-1}^{+,-}$and $\omega_{-1,+1}^{-,+}$

From

Table5: Vacuum Contractions (92),(93)

$\omega_{+1,-1}^{+,-}=$

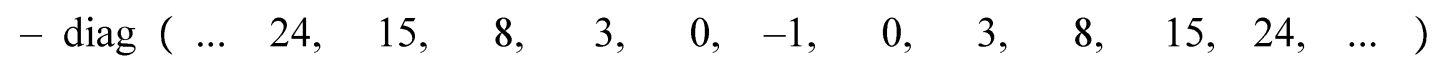
$\omega_{-1,+1}^{-,+}=$

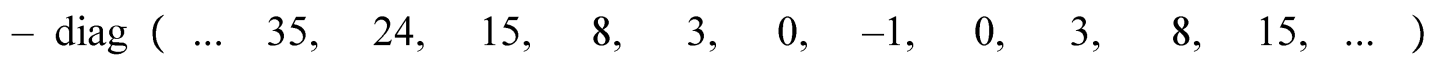

Obtain

Table6: $\omega_{1 ;+1,-1}^{2}+\omega_{2 ;+1,-1}^{2}$

$$
\omega_{1 ;+1,-1}^{2}+\omega_{2 ;+1,-1}^{2}=\frac{1}{2}\left(\omega_{+1,-1}^{+,-}+\omega_{-1,+1}^{-,+}\right)=
$$

$-\frac{1}{2} \operatorname{diag}(\ldots \quad 49, \quad 39, \quad 23, \quad 11, \quad 3,-1,-1, \quad 3, \quad 11, \quad 23, \quad 39, \quad \ldots \quad)$

$+\frac{1}{4} \operatorname{diag}(\ldots-98,-78,-46,-22,-6, \quad 2, \quad 2,-6,-22,-46,-78, \ldots)$

And

Table7: $\omega_{3 ;+1,-1}, \omega_{3 ;+1,-1}^{2}$

$\omega_{3 ;+1,-1}=\frac{1}{2}\left(\omega_{+1,-1}^{+,-}-\omega_{-1,+1}^{-,+}\right)=$

$+\frac{1}{2} \operatorname{diag}(\ldots \quad 11, \quad 9, \quad 7, \quad 5, \quad 3, \quad 1, \quad-1,-3, \quad-5, \quad-7, \quad-9, \quad \ldots \quad)$

$\omega_{3 ;+1,-1}^{2}=$

$+\frac{1}{4} \operatorname{diag}(\ldots 121, \quad 81, \quad 49, \quad 25, \quad 9, \quad 1, \quad 1, \quad 9, \quad 25, \quad 49, \quad 81, \quad \ldots \quad)$

Then

Table8: $\omega_{+1,-1}^{2}$

$$
\begin{aligned}
& \omega_{+1,-1}^{2}=\omega_{1 ;+1,-1}^{2}+\omega_{2 ;+1,-1}^{2}+\omega_{3 ;+1,-1}^{2}= \\
& +\frac{1}{4} \operatorname{diag}(\ldots 3, \quad 3, \quad 3, \quad 3, \quad 3, \quad 3, \quad 3, \quad 3, \quad 3, \quad 3, \quad 3, \quad \ldots \quad) \\
& =\frac{3}{4} I_{0}=\frac{3}{2}\left(\frac{3}{2}+1\right) I_{0}
\end{aligned}
$$

Table $7 \boldsymbol{\omega}_{3 ;+1,-1}$ and Table $8 \boldsymbol{\omega}_{+1,-1}^{2}$ are just the known $\hbar / 2$ fermion of CSH !

$$
\boldsymbol{\nabla} \omega_{+1,-1}^{2}=\Omega_{+1 / 2}^{2} \quad \omega_{3,+1,-1}=\Omega_{3,+1 / 2} \quad ! ! !
$$


6.3

Formation of $1 h$ bosons result in the third order Vacuum Bubble phase transition

by $\omega_{+2,-1}^{+,-}$and $\omega_{-1,+2}^{-,+}$

From

Table9: Vacuum Contractions (94),(95)

$\omega_{+2,-1}^{+,-}=$

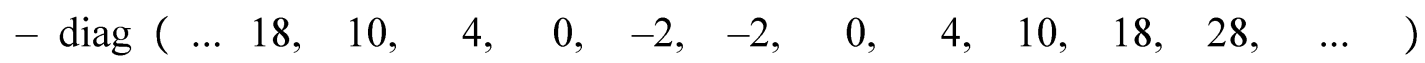
$\omega_{-1,+2}^{-,+}=$

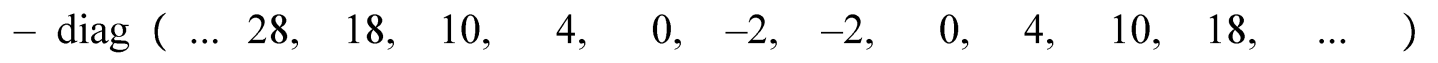

Obtain

Table10: $\omega_{1 ;+2,-1}^{2}+\omega_{2 ;+2,-1}^{2}$

$\omega_{1 ;+2,-1}^{2}+\omega_{2 ;+2,-1}^{2}=\frac{1}{2}\left(\omega_{+2,-1}^{+,-}+\omega_{-1,+2}^{-,+}\right)=$

$-\frac{1}{2} \operatorname{diag}(\ldots \quad 46, \quad 28,14,4,-2,-4,-2, \quad 4, \quad 14, \quad 28,46, \quad \ldots \quad)$

$\operatorname{diag}(\ldots-23,-14,-7,-2, \quad 1,2, \quad 1,-2,-7,-14,-23, \ldots)$

And

Table11: $\omega_{3 ;+2,-1}, \omega_{3 ;+2,-1}^{2}$

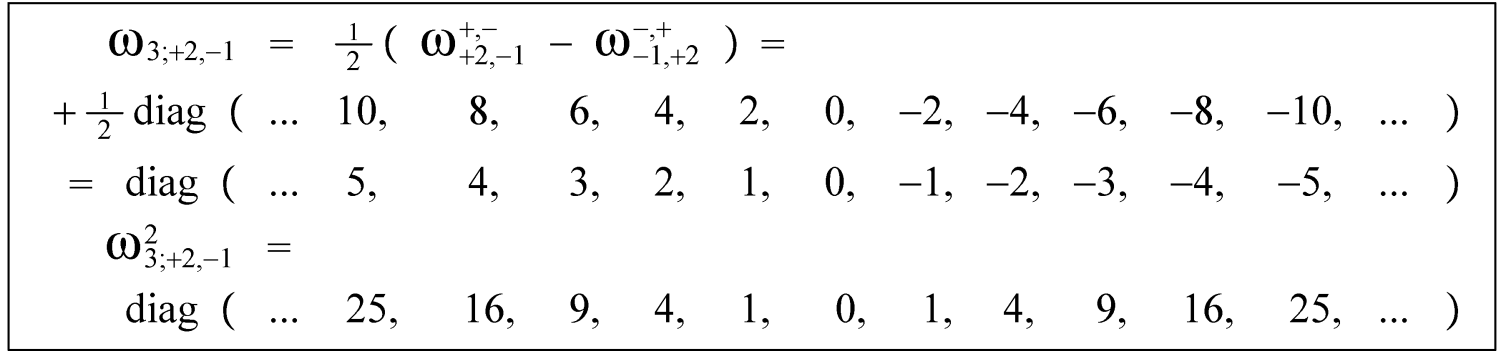

Then

Table12: $\omega_{+2,-1}^{2}$

$\omega_{+2,-1}^{2}=\omega_{1 ;+2,-1}^{2}+\omega_{2 ;+2,-1}^{2}+\omega_{3 ;+2,-1}^{2}=$

$\operatorname{diag}\left(\begin{array}{lllllllllllll}\ldots & 2, & 2, & 2, & 2, & 2, & 2, & 2, & 2, & 2, & 2, & 2, & \ldots\end{array}\right)$

$=2 I_{0}=1(1+1) I_{0}$

Table11 $\omega_{3 ;+2,-1}$ and Table12 $\omega_{+2,-1}^{2}$ are just the known $1 \hbar$ boson of CSH !

$$
\text { A } \omega_{+2,-1}^{2}=\Omega_{+1}^{2} \quad \omega_{3,+2,-1}=\Omega_{3,+1} \quad \text { !!! }
$$


6.4

Formation of $3 h / 2$ fermions result in the fourth order Vacuum Bubble phase transition by $\omega_{+2,-2}^{+,-}$and $\omega_{-2,+2}^{-,+}$

From

Table13: Vacuum Contractions (96),(97)

$\omega_{+2,-2}^{+,-}=$

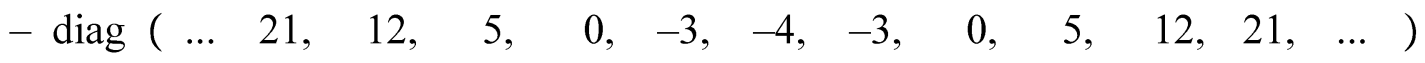
$\omega_{-2,+2}^{-++}=$

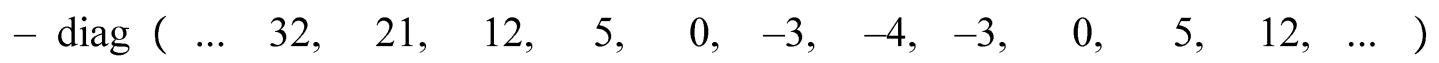

Obtain

Table14: $\omega_{1 ;+2,-2}^{2}+\omega_{2 ;+2,-2}^{2}$

$\omega_{1 ;+2,-2}^{2}+\omega_{2 ;+2,-2}^{2}=\frac{1}{2}\left(\omega_{+2,-2}^{+,-}+\omega_{-2,+2}^{-,+}\right)=$

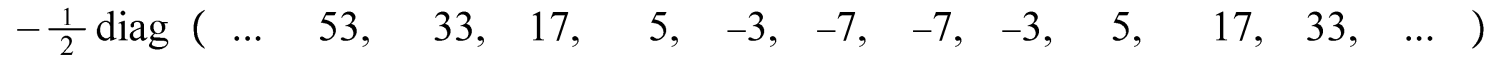

$+\frac{1}{4} \operatorname{diag}(\ldots-106,-66,-34,-10,6, \quad 14, \quad 14,6,-10,-34,-66, \ldots)$

And

Table15: $\omega_{3 ;+2,-2}, \omega_{3 ;+2,-2}^{2}$

$\omega_{3 ;+2,-2}=\frac{1}{2}\left(\omega_{+2,-2}^{+,-}-\omega_{-2,+2}^{-,+}\right)=$

$+\frac{1}{2} \operatorname{diag}(\ldots \quad 11, \quad 9, \quad 7, \quad 5, \quad 3, \quad 1, \quad-1,-3, \quad-5, \quad-7, \quad-9, \quad \ldots \quad)$

$\omega_{3 ;+2,-2}^{2}=$

$+\frac{1}{4} \operatorname{diag}(\ldots 121, \quad 81, \quad 49, \quad 25, \quad 9, \quad 1, \quad 1, \quad 9, \quad 25, \quad 49, \quad 81, \quad \ldots \quad)$

Then

Table16: $\omega_{+2,-2}^{2}$

$\omega_{+2,-2}^{2}=\omega_{1 ;+2,-2}^{2}+\omega_{2 ;+2,-2}^{2}+\omega_{3 ;+2,-2}^{2}=$

$+\frac{1}{4} \operatorname{diag}(\ldots 15,15,15,15,15,15,15,15,15,15,15, \ldots)$

$=\frac{15}{4} I_{0}=\frac{3}{2}\left(\frac{3}{2}+1\right) I_{0}$

Table15 $\omega_{3 ;+2,-2}$ and Table16 $\omega_{+2,-2}^{2}$ are just the known $3 \hbar / 2$ fermion of CSH !

$$
\omega_{+2,-2}^{2}=\Omega_{+3 / 2}^{2} \quad \omega_{3,+2,-2}=\Omega_{3,+3 / 2} \quad \text { !!! }
$$




\subsection{Concluding Summations}

- Table17: Vacuum Spin Particle, VSP with spin $-\frac{1}{2}$ formed from 0 th order phase transition by $\omega_{0,0}^{+,-}$and $\omega_{0,0}^{-,+} . \quad \omega_{0,0}^{2}=-\frac{1}{2}\left(-\frac{1}{2}+1\right)=-\frac{1}{4}$

$\omega_{3 ; 0,0}$

$=\operatorname{diag}(\ldots 11 / 2,9 / 2,7 / 2,5 / 2,3 / 2,1 / 2,-1 / 2,-3 / 2,-5 / 2,-7 / 2,-9 / 2, \ldots)$

$\Delta$ Table18: $0 h$ bosons formed from 1st order phase transition by $\omega_{+1,0}^{+,-}$and $\omega_{0,+1}^{-,+}: \quad \omega_{+1,0}^{2}=0(0+1)=0$

$\omega_{3 ;+1,0}$

$=\operatorname{diag}(\ldots \quad 5, \quad 4, \quad 3, \quad 2, \quad 1, \quad 0, \quad-1, \quad-2, \quad-3, \quad-4, \quad-5, \quad \ldots)$

$\nabla$ Table19: $\hbar / 2$ fermions formed from 2 nd order phase transition by $\omega_{+1,-1}^{+,-}$and $\omega_{-1,+1}^{-,+}: \omega_{+1,-1}^{2}=+\frac{1}{2}\left(+\frac{1}{2}+1\right)=\frac{3}{4}$

$\omega_{3 ;+1,-1}$

$=\operatorname{diag}(\ldots 11 / 2,9 / 2,7 / 2,5 / 2,3 / 2,1 / 2,-1 / 2,-3 / 2,-5 / 2,-7 / 2,-9 / 2, \ldots)$

A Table20: $1 \hbar$ bosons formed from 3rd order phase transition by $\omega_{+2,-1}^{+,-}$and $\omega_{-1,+2}^{-,+}: \omega_{+2,-1}^{2}=1(1+2)=2$

$\omega_{3 ;+2,-1}$

$=\operatorname{diag}(\ldots \quad 5, \quad 4, \quad 3, \quad 2, \quad 1, \quad 0, \quad-1, \quad-2, \quad-3, \quad-4, \quad-5, \quad \ldots)$

- Table21: $3 \hbar / 2$ fermions formed from 4th order phase transition by $\omega_{+2,-2}^{+,-}$and $\omega_{-2,+2}^{-,+}: \quad \omega_{+2,-2}^{2}=\frac{3}{2}\left(\frac{3}{2}+1\right)=\frac{15}{4}$

$\omega_{3 ;+2,-2}$

$=\operatorname{diag}(\ldots 11 / 2,9 / 2,7 / 2,5 / 2,3 / 2,1 / 2,-1 / 2,-3 / 2,-5 / 2,-7 / 2,-9 / 2, \ldots)$ 
7. Vacuum Bubble Pair VBP $\omega_{\mathrm{S}=|i-j|}^{+,-}(k)$ of the $k$ th generations of CSH

In phase transition processes, contraction $\omega_{i, j}^{+,-}$and contraction $\omega_{j, i}^{-,+}$ always appear to pair up together, then a mapping called Vacuum Bubble Pair VBP is introduced:

$$
\begin{aligned}
\omega_{i, j}^{+,-}(k) \text { and } \omega_{j, i}^{-,+}(k) & \rightarrow\left\{\omega_{i, j}^{+,-}(k), \omega_{j, i}^{-,+}(k)\right\} \in \omega_{\mathrm{S}=|i-j|}^{+,-}(k): \quad \mathbf{V B P} \\
\mathrm{S} & =|i-j| ; \quad k=1,2,3, \ldots
\end{aligned}
$$

Where $\omega_{\mathrm{S}=|i-j|}^{+,-}(k)$ are the sets used to represent

$$
\omega_{\mathrm{S}=|i-j|}^{+,-}(k) \text { : the sth order phase transition of Vacuum Bubble Pair }
$$

In the previous discussions:

a) Mapping (106) indicates Self-identical bubble Vacuum contractions $\omega^{+,-}(73), \omega^{-,+}(74)$ or $\omega_{0,0}^{+,-}(86), \omega_{0,0}^{-,+}(87) \quad \in \omega_{\mathrm{S}=0}^{+,-}(k=1)$.

b) Mapping (107)-(110) indicate the four groups of Dual-identical bubble Vacuum contractions $\omega_{+1,0}^{+,-}(90), \omega_{0,+1}^{-,+}(91) ; \omega_{+1,-1}^{+,-}(92), \omega_{-1,+1}^{-,+}(93) ; \omega_{+2,-1}^{+,-}(94)$, $\omega_{-1,+2}^{-,+}(95) ; \omega_{+2,-2}^{+,-}(96), \omega_{-2,+2}^{-,+}(97) \in \omega_{\mathrm{S} \neq 0}^{+,-}(k=1)$.

In above paragraph 6. what follows mean:

$$
\begin{aligned}
& \omega_{0,0}^{+,-}, \omega_{0,0}^{-,+}(86),(87) \rightarrow \quad\left\{\omega_{0,0}^{+,-}(1), \omega_{0,0}^{-,+}(1)\right\} \quad \in \omega_{\mathrm{S}=0}^{+,-}(1) \\
& \omega_{+1,0}^{+,-}, \omega_{0,+1}^{-,+} \quad \mathbf{6 . 1} \rightarrow\left\{\omega_{+1,0}^{+,-}(1), \omega_{0,+1}^{-,+}(1)\right\} \in \omega_{\mathrm{S}=1}^{+,-}(1) \\
& \omega_{+1,-1}^{+,-}, \omega_{-1,+1}^{-,+} \mathbf{6 . 2} \rightarrow\left\{\omega_{+1,-1}^{+,-}(1), \omega_{-1,+1}^{-,+}(1)\right\} \quad \in \omega_{\mathrm{S}=2}^{+,-}(1) \\
& \omega_{+2,-1}^{+,-}, \omega_{-1,+2}^{-,+} 6.3 \rightarrow\left\{\omega_{+2,-1}^{+,-}(1), \omega_{-1,+2}^{-,+}(1)\right\} \quad \in \omega_{\mathrm{S}=3}^{+,-}(1) \\
& \omega_{+2,-2}^{+,-}, \omega_{-2,+2}^{-,+} 6.4 \rightarrow\left\{\omega_{+2,-2}^{+,-}(1), \omega_{-2,+2}^{-,+}(1)\right\} \in \omega_{\mathrm{S}=4}^{+,-}(1)
\end{aligned}
$$

In following paragraph 8. what follows will be used:

$$
\begin{array}{r}
\ldots\left\{\omega_{-1,-1}^{+,-}(2), \omega_{-1,-1}^{-,+}(2)\right\} ;\left\{\omega_{0,0}^{+,-}(2), \omega_{0,0}^{-,+}(2)\right\} ;\left\{\omega_{+1,+1}^{+,-}(2), \omega_{+1,+1}^{-,+}(2)\right\} \ldots \in \omega_{\mathrm{S}=0}^{+,-}(2) \\
\ldots\left\{\omega_{0,-1}^{+,-}(2), \omega_{-1,0}^{-,+}(2)\right\} ;\left\{\omega_{+1,0}^{+,-}(2), \omega_{0,+1}^{-,+}(2)\right\} ;\left\{\omega_{+2,+1}^{+,-}(2), \omega_{+1,+2}^{-,+}(2)\right\} \ldots \in \omega_{\mathrm{S}=1}^{+,-}(2) \\
\ldots\left\{\omega_{+1,-1}^{+,-}(2), \omega_{-1,+1}^{-,+}(2)\right\} ;\left\{\omega_{+2,0}^{+,-}(2), \omega_{0,+2}^{-,+}(2)\right\} ;\left\{\omega_{+3,+1}^{+,-}(2), \omega_{+1,+3}^{-,+}(2)\right\} \ldots \in \omega_{\mathrm{S}=2}^{+,-}(2) \\
\ldots \in \omega_{\mathrm{S}=3}^{+,-}(2)
\end{array}
$$

In following paragraph 9. what follows will be used:

$$
\begin{array}{r}
\ldots\left\{\omega_{-1,-1}^{+,-}(3), \omega_{-1,-1}^{-,+}(3)\right\} ;\left\{\omega_{0,0}^{+,-}(3), \omega_{0,0}^{-,+}(3)\right\} ;\left\{\omega_{+1,+1}^{+,-}(3), \omega_{+1,+1}^{-,+}(3)\right\} \ldots \in \\
\ldots\left\{\omega_{0,-1}^{+,-}(3), \omega_{-1,0}^{-,+}(3)\right\} ;\left\{\omega_{+1,0}^{+,-}(3), \omega_{0,+1}^{-,+}(3)\right\} ;\left\{\omega_{+2,+1}^{+,-}(3), \omega_{+1,+2}^{-,+}(3)\right\} \ldots \in \omega_{\mathrm{S}=1}^{+,-}(3) \\
\ldots\left\{\omega_{+1,-1}^{+,-}(3), \omega_{-1,+1}^{-,+}(3)\right\} ;\left\{\omega_{+2,0}^{+,-}(3), \omega_{0,+2}^{-,+}(3)\right\} ;\left\{\omega_{+3,+1}^{+,-}(3), \omega_{+1,+3}^{-,+}(3)\right\} \ldots \in \omega_{\mathrm{S}=2}^{+,-}(3) \\
\ldots \in \omega_{\mathrm{S}=3}^{+,-}(3)
\end{array}
$$


8. Formation of bosons, fermions and TKP and VSP

of the second generation of $\mathbf{C S H}$

Table22: Primitive Spin Particle, VSP with spin $-\frac{1}{2}$

formed from 0th order phase transition

of the second generation of CSH

by $\omega_{\mathrm{S}=0}^{+,-}(2): \quad \omega_{\mathrm{S}=0}^{2}(2)=-\frac{1}{2}\left(-\frac{1}{2}+1\right)=-\frac{1}{4}$

$\omega_{3, \mathrm{~S}=0}^{+,-}(2)$

$=\operatorname{diag}(\ldots \quad 3, \quad 5 / 2, \quad 2, \quad 3 / 2, \quad 1, \quad 1 / 2, \quad 0, \quad-1 / 2, \quad-1, \quad-3 / 2, \quad-2, \quad \ldots)$

Table23: $-\frac{1}{4}$ spin TKP

formed from 1st order phase transition

of the second generation of CSH

by $\omega_{\mathrm{S}=1}^{+,-}(2): \quad \omega_{\mathrm{S}=1}^{2}(2)=-\frac{1}{4}\left(-\frac{1}{4}+1\right)=-\frac{3}{16}$

$\omega_{3, \mathrm{~S}=1}^{+,-}(2)$

$=\operatorname{diag}(\ldots 11 / 4,9 / 4,7 / 4, \quad 5 / 4,3 / 4, \quad 1 / 4,-1 / 4,-3 / 4,-5 / 4,-7 / 4,-9 / 4, \ldots)$

Table24: $0 h$ bosons formed from 2 nd order phase transition of the second generation of $\mathbf{C S H}$

by $\omega_{\mathrm{S}=2}^{+,-}(2): \quad \omega_{\mathrm{S}=2}^{2}(2)=0(0+1)=0$

$\omega_{3, \mathrm{~s}=2}^{+,-}(2)$

$=\operatorname{diag}(\ldots \quad 3, \quad 5 / 2, \quad 2, \quad 3 / 2, \quad 1, \quad 1 / 2, \quad 0, \quad-1 / 2, \quad-1, \quad-3 / 2, \quad-2, \quad \ldots)$

Table25: $\hbar / 4$ TKP formed from 3rd order phase transition of the second generation of CSH

by $\omega_{\mathrm{S}=3}^{+,-}(2): \quad \omega_{\mathrm{S}=3}^{2}(2)=\frac{1}{4}\left(\frac{1}{4}+1\right)=\frac{5}{16}$

$\omega_{3, \mathrm{~s}=3}^{+,-}(2)$

$=\operatorname{diag}(\ldots 11 / 4,9 / 4,7 / 4,5 / 4,3 / 4, \quad 1 / 4,-1 / 4,-3 / 4,-5 / 4,-7 / 4,-9 / 4, \ldots)$

Table26: $\hbar / 2$ fermions formed from 4 th order phase transition of the second generation of CSH

by $\omega_{\mathrm{S}=4}^{+,-}(2): \quad \omega_{\mathrm{S}=4}^{2}(2)=\frac{1}{2}\left(\frac{1}{2}+1\right)=\frac{3}{4}$

$\omega_{3, \mathrm{~S}=4}^{+,-}(2)$

$=\operatorname{diag}(\ldots \quad 3, \quad 5 / 2, \quad 2, \quad 3 / 2, \quad 1, \quad 1 / 2, \quad 0, \quad-1 / 2, \quad-1, \quad-3 / 2, \quad-2, \quad \ldots)$ 
9. Formation of bosons, fermions and TKP and VSP

of the third generation of $\mathbf{C S H}$

Table27: Vacuum Spin Particle, VSP with spin $-\frac{1}{2}$

formed from 0th order phase transition of the third generation of CSH

by $\omega_{\mathrm{S}=0}^{+,-}(3): \quad \omega_{\mathrm{S}=0}^{2}(3)=-\frac{1}{2}\left(-\frac{1}{2}+1\right)=-\frac{1}{4}$

$\omega_{3, \mathrm{~s}=0}^{+,-}(3)$

$=\operatorname{diag}(\ldots 11 / 6, \quad 3 / 2, \quad 7 / 6, \quad 5 / 6, \quad 1 / 2, \quad 1 / 6,-1 / 6,-1 / 2,-5 / 6,-7 / 6,-3 / 2, \ldots)$

Table28: $-\frac{1}{3}$ spin TKP

formed from 1 st order phase transition

of the third generation of $\mathbf{C S H}$

by $\omega_{\mathrm{S}=1}^{+,-}(3): \quad \omega_{\mathrm{S}=1}^{2}(3)=-\frac{1}{3}\left(-\frac{1}{3}+1\right)=-\frac{2}{9}$

$\omega_{3, \mathrm{~s}=1}^{+,-}(3)$

$=\operatorname{diag}(\ldots 5 / 3, \quad 4 / 3, \quad 1, \quad 2 / 3, \quad 1 / 3, \quad 0, \quad-1 / 3,-2 / 3, \quad-1, \quad-4 / 3,-5 / 3, \ldots)$

Table29: $-\frac{1}{6}$ spin TKP

formed from 2 nd order phase transition

of the third generation of $\mathbf{C S H}$

by $\omega_{\mathrm{S}=2}^{+,-}(3): \quad \omega_{\mathrm{S}=2}^{2}(3)=-\frac{1}{6}\left(-\frac{1}{6}+1\right)=-\frac{5}{36}$

$\omega_{3, \mathrm{~s}=2}^{+,-}(3)$

$=\operatorname{diag}(\ldots 11 / 6, \quad 3 / 2, \quad 7 / 6, \quad 5 / 6, \quad 1 / 2, \quad 1 / 6,-1 / 6,-1 / 2,-5 / 6, \quad-7 / 6,-3 / 2, \ldots)$

Table 30: $0 h$ bosons formed from 3rd order phase transition of the third generation of CSH

by $\omega_{\mathrm{S}=3}^{+,-}(3): \quad \omega_{\mathrm{S}=3}^{2}(3)=0(0+1)=0$

$\omega_{3, \mathrm{~s}=3}^{+,-}(3)$

$=\operatorname{diag}(\ldots \quad 2, \quad 5 / 3, \quad 4 / 3, \quad 1, \quad 2 / 3, \quad 1 / 3, \quad 0, \quad-1 / 3,-2 / 3, \quad-1, \quad-4 / 3, \ldots)$ 
Table31: $\hbar / 6$ TKP formed from 4th order phase transition of the third generation of CSH

$$
\text { by } \omega_{\mathrm{S}=4}^{+,-}(3): \quad \omega_{\mathrm{S}=4}^{2}(3)=\frac{1}{6}\left(\frac{1}{6}+1\right)=\frac{7}{36}
$$

$\omega_{3, \mathrm{~s}=4}^{+,-}(3)$

$=\operatorname{diag}(\ldots 11 / 6, \quad 3 / 2, \quad 7 / 6, \quad 5 / 6, \quad 1 / 2, \quad 1 / 6, \quad-1 / 6,-1 / 2,-5 / 6,-7 / 6,-3 / 2, \ldots)$

Table32: $\hbar / 3$ TKP formed from 5th order phase transition of the third generation of $\mathbf{C S H}$

$$
\text { by } \omega_{\mathrm{S}=5}^{+,-}(3): \quad \omega_{\mathrm{S}=5}^{2}(3)=\frac{1}{3}\left(\frac{1}{3}+1\right)=\frac{4}{9}
$$

$\omega_{3,-}^{+,-}=5(3)$

$=\operatorname{diag}(\ldots \quad 5 / 3,4 / 3, \quad 1, \quad 2 / 3, \quad 1 / 3, \quad 0, \quad-1 / 3,-2 / 3, \quad-1, \quad-4 / 3,-5 / 3, \ldots)$

Table33: $\hbar / 2$ fermions formed from 6 th order phase transition of the third generation of $\mathbf{C S H}$

by $\omega_{\mathrm{S}=6}^{+,-}(3): \quad \omega_{\mathrm{S}=6}^{2}(3)=\frac{1}{2}\left(\frac{1}{2}+1\right)=\frac{3}{4}$

$\omega_{3, \mathrm{~s}=6}^{+,-}(3)$

$=\operatorname{diag}(\ldots 11 / 6, \quad 3 / 2, \quad 7 / 6, \quad 5 / 6, \quad 1 / 2, \quad 1 / 6, \quad-1 / 6,-1 / 2,-5 / 6,-7 / 6,-3 / 2, \ldots)$

Table34: $2 \hbar / 3$ TKP formed from 7 th order phase transition of the third generation of $\mathbf{C S H}$

by $\omega_{\mathrm{S}=7}^{+,-}(3): \quad \omega_{\mathrm{S}=7}^{2}(3)=\frac{2}{3}\left(\frac{2}{3}+1\right)=\frac{10}{9}$

$\omega_{3, \mathrm{~s}=7}^{+,-}(3)$

$=\operatorname{diag}(\ldots \quad 5 / 3,4 / 3, \quad 1, \quad 2 / 3, \quad 1 / 3, \quad 0, \quad-1 / 3,-2 / 3, \quad-1, \quad-4 / 3,-5 / 3, \ldots)$

Table35: $5 \hbar / 6$ TKP formed from 8 th order phase transition of the third generation of $\mathbf{C S H}$

by $\omega_{\mathrm{S}=8}^{+,-}(3): \quad \omega_{\mathrm{S}=8}^{2}(3)=\frac{5}{6}\left(\frac{5}{6}+1\right)=\frac{55}{36}$

$\omega_{3, \mathrm{~S}=8}^{+,-}(3)$

$=\operatorname{diag}(\ldots 11 / 6, \quad 3 / 2, \quad 7 / 6, \quad 5 / 6, \quad 1 / 2, \quad 1 / 6, \quad-1 / 6,-1 / 2,-5 / 6,-7 / 6,-3 / 2, \quad \ldots)$ 
10. The sth order phase transitions of Vacuum Bubble Pair $\omega_{\mathrm{S}=|i-j|}^{+,-}(k)$ in SAMV ocean.

The results of paragraphs 6., 7., 8., 9. are tabled as below.

Table36 Contributions of Vacuum Bubble Pair VBP to Phase Transition (PT ) for the 1st, 2nd and 3rd of CSH

\begin{tabular}{|c|c|c|c|c|c|c|}
\hline VBP & $1 \mathrm{st}$ & CSH & 2nd & CSH & $3 r d$ & CSH \\
\hline$\omega_{\mathrm{S}}^{+,-}(k)$ & $\omega_{\mathrm{S}}^{2}(1)$ & $\operatorname{Spin}(1)$ & $\omega_{\mathrm{S}}^{2}(2)$ & $\operatorname{Spin}(2)$ & $\omega_{S}^{2}(3)$ & $\operatorname{Spin}(3)$ \\
\hline$\omega_{\mathrm{S}=8}^{+,-}$ & $\frac{63}{4}-$ & $-\quad \frac{7}{2}$ & $\frac{15}{4}=\frac{60}{16}$ & $-\frac{6}{4}=\frac{3}{2}$ & $\frac{55}{36}$ & $-\frac{5}{6}$ \\
\hline$\omega_{\mathrm{S}=7}^{+,-}$ & $12=\frac{48}{4}$ & $\frac{6}{2}=3$ & $\frac{45}{16}$ & $\frac{5}{4}$ & $\frac{10}{9}=\frac{40}{36}$. & $-\frac{4}{6}=\frac{2}{3}$ \\
\hline$\omega_{\mathrm{S}=6}^{+,-}$ & $\frac{35}{4}$ & $\frac{5}{2}$ & $2=\frac{32}{16}$ & $\frac{4}{4}=1$ & $\frac{3}{4}=\frac{27}{36}$ & $\frac{3}{6}=\frac{1}{2}$ \\
\hline$\omega_{\mathrm{S}=5}^{+,-}$ & $6=\frac{24}{4}-$ & $\frac{4}{2}=2$ & $\frac{21}{16}$ & $\frac{3}{4}$ & $\frac{4}{9}=\frac{16}{36}$ & $\frac{2}{6}=\frac{1}{3}$ \\
\hline$\omega_{\mathrm{S}=4}^{+,-}$ & $\frac{15}{4}$ & $-\frac{3}{2}$ & $\frac{3}{4}=\frac{12}{16}$ & $\frac{2}{4}=\frac{1}{2}$ & $\frac{7}{36}$ & $\frac{1}{6}$ \\
\hline$\omega_{\mathrm{S}=3}^{+,-}$ & $2=\frac{8}{4}$ & $-\frac{2}{2}=1$ & $\frac{5}{16}$ & $\frac{1}{4}$ & $0=\frac{\mathbf{0}}{36}$ & $\frac{0}{6}=0$ \\
\hline$\omega_{\mathrm{S}=2}^{+,-}$ & $\frac{3}{4}$ & $-\nabla-\frac{1}{2}$ & $0=\frac{\mathbf{0}}{16}$ & $\frac{0}{4}=0$ & $-\frac{5}{36}$ & $--\frac{1}{6}$ \\
\hline$\omega_{\mathrm{S}=1}^{+,-}$ & $0=\frac{\mathbf{0}}{4}$ & $-\boldsymbol{\Delta}-\frac{0}{2}=0$ & $-\frac{3}{16}$ & $--\frac{1}{4}$ & $-\frac{2}{9}=-\frac{8}{36}$ & $--\frac{2}{6}=-\frac{1}{3}$ \\
\hline$\omega_{\mathrm{S}=0}^{+,-}$ & $-\frac{1}{4}$ & $-\frac{1}{2}$ & $-\frac{4}{16}$ & $--\frac{2}{4}$ & $-\frac{9}{36}$ & $1-\frac{3}{6}$ \\
\hline$\omega_{\mathrm{S}=0}^{+,-}$ & $-\frac{1}{4}-$ & $--\frac{1}{2}$ & $-\frac{1}{4}$ & $--\frac{1}{2}$ & $-\frac{1}{4}$ & $--\frac{1}{2}$ \\
\hline$\omega_{\mathrm{S}}^{+,-}(k)$ & $\omega_{\mathrm{S}}^{2}(1)$ & $\operatorname{Spin}(1)$ & $\omega_{\mathrm{S}}^{2}(2)$ & $\operatorname{Spin}(2)$ & $\omega_{\mathrm{S}}^{2}(3)$ & $\operatorname{Spin}(3)$ \\
\hline VBP & $1 \mathrm{st}$ & CSH & 2nd & CSH & $3 \mathrm{rd}$ & CSH \\
\hline
\end{tabular}


11. Spin particles with Casimir operator $\omega_{\mathrm{S}}^{2}$

formed of Vacuum Bubble Pair VBP $\omega_{\mathrm{S}}^{+}$

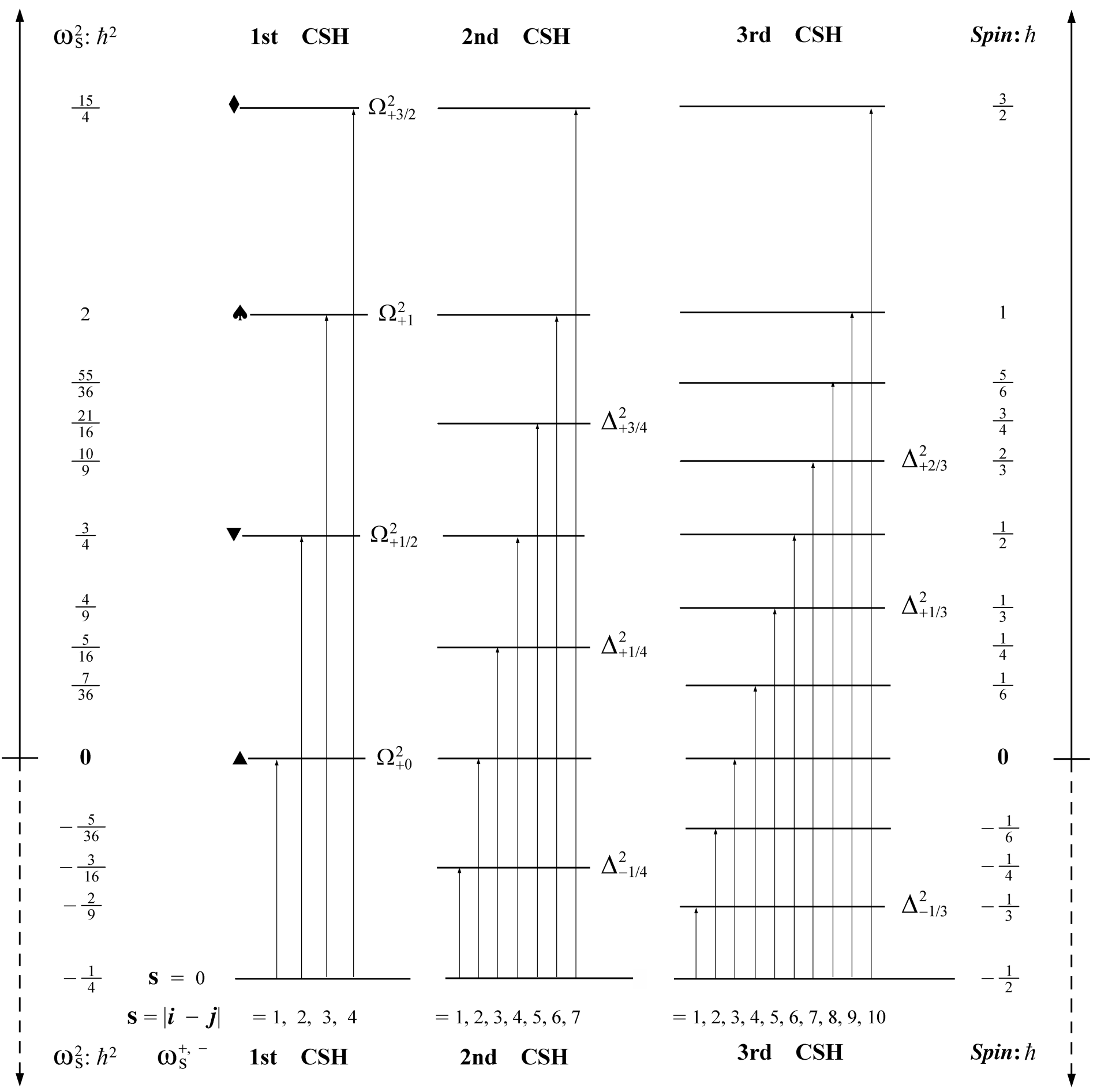

FIG.2. Phase transitions of Vacuum Bubble Pair $\omega_{\mathrm{S}=|i-j|}^{+,-}(k)$ from the sea floor one-fourth $-\frac{1}{4} \hbar^{2}$ deep in SAMV ocean (not to scale) 
12. Local and Nonlocal Spin Angular Momentum Commutation Rules In conventional quantum mechanics, spin particles obey

$$
\begin{aligned}
& {\left[\mathrm{s}_{i, x}, \mathrm{~s}_{i, y}\right]_{-}=i \mathrm{~s}_{i, z}, \quad x, y, z \text { circulative }} \\
& {\left[\mathrm{s}_{j, x}, \mathrm{~s}_{j, y}\right]_{-}=i \mathrm{~s}_{j, z},} \\
& {\left[\mathrm{~s}_{i, x}, \mathrm{~s}_{j, y}\right]_{-}=0, \quad i \neq i \text { for any } x, y, z}
\end{aligned}
$$

$\mathrm{s}_{i, x}, \mathrm{~s}_{j y}$ indicate $x$ component of particle $i, y$ component of particle $j$.

We call above commutation rules (119) Local Spin Particle Angular Momentum Commutation Rules LR

The commutation rules (60), of Spin $-\frac{1}{2} \hbar$ VSP particle what we discussed in paragraph $\mathbf{3}$, are $\mathbf{L R}$.

LR are connected to Self-identical bubble vacuum contractions.

Using (64),(65) then (119) can be written as (120) below:

1) Local commutations, only for single particle $i$ itself

$$
\begin{aligned}
\mathrm{s}_{j, 3} \mathrm{~s}_{j}^{+}-\mathrm{s}_{j}^{+} \mathrm{s}_{j, 3} & =+\mathrm{s}_{j}^{+} \\
\mathrm{s}_{j, 3} \mathrm{~s}_{j}^{-}-\mathrm{s}_{j}^{-} \mathrm{s}_{j, 3} & =-\mathrm{s}_{j}^{-} \\
\mathrm{s}_{j}^{+} \mathrm{s}_{j}^{-}-\mathrm{s}_{j}^{-} \mathrm{s}_{j}^{+} & =2 \mathrm{~s}_{j, 3}^{+}
\end{aligned}
$$

2) Non-local commutations, for both particle $i$ and particle $j$

$$
\begin{aligned}
& \mathrm{S}_{i j, 3} \mathrm{~S}_{i}^{+}-\mathrm{S}_{i}^{+} \mathrm{S}_{i j, 3}=0 \\
& \mathrm{~s}_{i j, 3} \mathrm{~S}_{j}^{-}-\mathrm{S}_{j}^{-} \mathrm{S}_{i j, 3}=0 \\
& \mathrm{~s}_{i}^{+} \mathrm{s}_{j}^{-}-\mathrm{s}_{j}^{-} \mathrm{s}_{i}^{+}=0
\end{aligned}
$$

The appearance of Dual-identical bubble vacuum contractions, lead to a new concept called Nonlocality of Vacuum Contractions.

(120) Locality:

$$
\omega_{j}^{+} \omega_{j}^{-}-\omega_{j}^{-} \omega_{j}^{+}=\omega_{j, j}^{+,-}-\omega_{j, j}^{-,+} \neq 0
$$

(121) NonLocality:

$$
\omega_{i}^{+} \omega_{j}^{-}-\omega_{j}^{-} \omega_{i}^{+}=\omega_{i, j}^{+,-}-\omega_{j, i}^{-,+} \neq 0
$$

And commutation rules (121) turn into

$$
\begin{aligned}
\omega_{3} \omega_{i}^{+}-\omega_{i}^{+} \omega_{3} & =+\omega_{i}^{+} \\
\omega_{3} \omega_{j}^{-}-\omega_{j}^{-} \omega_{3} & =-\omega_{j}^{-} \\
\omega_{i}^{+} \omega_{j}^{-}-\omega_{j}^{-} \omega_{i}^{+} & =2 \omega_{3} \\
i & \neq j
\end{aligned}
$$


(124) are called NonLocal Spin Angular Momentum Commutation Rules or NLR.

When $i=j$, NLR degenerate to LR.

The more detailed proofs of VSP NLR are given in cite [3].

\section{Conclusions}

Bosons and Fermions are the offspring of Vacuum Spin Particles. VSP.

In this paper, VSP is $-\frac{1}{2} \hbar$ spin particle, labeled by $\omega_{j}=\Omega_{j,-1 / 2}=\omega_{j, \mathrm{~S}=0}(k)$, which lie on the sea floor of the shallow water region $\left(-\frac{1}{4} \hbar^{2}\right.$ deep) in Spin Angular Momentum Vacuum SAMV ocean.

By means of Matrix Product $\omega^{+} \omega^{-}$and $\omega^{-} \omega^{+}$, named Vacuum Contraction VC of Vacuum Spin Particles $\boldsymbol{\omega}_{j}$, then Vacuum Bubbles VB (see 4.) $\omega^{+-}$and $\omega^{-+}$are formed.

VB are identical bubbles. which could provide all types of Vacuum Bubble Pair VBP $\omega_{\mathrm{S}}^{+,}(k)$ (see 7.)

There are nany body interactions between these VBP, Such as Self-identical vacuum bubble interaction (see 4.2), Dual-identical vacuum bubble interaction (see $\mathbf{4 . 3}$ ),...

VSP $\omega_{j}\left(\right.$ or $\left.\omega^{+}, \omega^{-}\right)$results from Self-identical vacuum bubble interaction $\omega_{\mathrm{S}=0}^{+,-}(k)$ through the zero order Phase Transition PT.

All known Bosons and all known Fermions result from Dual-identical vacuum bubble interaction $\omega_{\mathrm{S} \neq 0}^{+,-}(k)$ through mult-order Phase Transitions PT (see 7., 6., 8., 9.), which are shown in Table36, Table37 and FIG.

Table37

$$
\begin{aligned}
& k=1,2,3, \ldots \omega_{\mathrm{S}}^{2}(k) \quad \text { Spin } \\
& \omega_{\mathrm{S}=4}^{+,-}(k) \quad \frac{15}{4} \longrightarrow \frac{3}{2}=\Omega_{+3 / 2}^{+,-} \in \omega_{\mathrm{S}=4}^{+,-}(k) \\
& \omega_{\mathrm{S}=3}^{+,-}(k) \quad 2 \longrightarrow \quad 1=\Omega_{+1}^{+,-} \in \omega_{\mathrm{S}=3}^{+,-}(k) \\
& \omega_{\mathrm{S}=2}^{+,-}(k) \quad \frac{3}{4} \longrightarrow \frac{1}{2}=\Omega_{+1 / 2}^{+,-} \in \omega_{\mathrm{S}=2}^{+,-}(k) \\
& \omega_{\mathrm{S}=1}^{+,-}(k) \quad 0 \longrightarrow \quad 0=\Omega_{+0}^{+,-} \in \omega_{\mathrm{S}=1}^{+,-}(k) \\
& \omega_{\mathrm{S}=0}^{+,-}(k) \quad-\frac{1}{4}--\frac{1}{2}=\Omega_{-1 / 2}^{+,-} \in \omega_{\mathrm{S}=0}^{+,-}(k)
\end{aligned}
$$


Where

$$
\begin{aligned}
\Omega_{j}^{+} & =\Omega_{1, j}+i \Omega_{2, j} \\
\Omega_{j}^{-} & =\Omega_{1, j}-i \Omega_{2, j} \\
j & =+0,+1 / 2,+1,+3 / 2, \ldots \\
\Omega_{j}^{+,-} & =\Omega_{j}^{+} \Omega_{j}^{-} \\
\Omega_{j}^{-,+} & =\Omega_{j}^{-} \Omega_{j}^{+}
\end{aligned}
$$

The physical picture of Vacuum Bubbles VB $\omega^{+,-}$and $\omega^{-,+}$are described to be "sub-observable physical quantities", because they are "diagonalized". But not any reality of today's quantum world are corresponding to them.

The principle of Vacuum Bubble Pair VBP $\omega_{\mathrm{S}=|i-j|}^{+,-}(k)$ is the key figure in the formations of bosons, fermions and all other potential spin particles that predicted.

VSP, $-\frac{1}{2} \hbar$ spin particle is the "critical particle" of Primitive Spin Particles PSP, if the deepth of SAMV ocean is greater than $\omega_{S}^{2}=-\frac{1}{4} \hbar^{2}$, Primitive Spin Particles PSP would bocome the imaginary number or complex number spin particles. how about the physical pictures of them ?

The fundamental nature of them will become more mysterious, of course.

This paper explains how well-known Bosons and well-known Fermions in universe are constructed from more fundamental matter spin particles. And postulates the existence of VSP, $-\frac{1}{2} \hbar$ spin particle.

If reasonable, more or less, the detection of TKP seems to be the priority of all...

Hope the idea of this paper would be beneficial to the fellows of physics and Math.

This paper is the selections of cite: [3]

\section{References}

[1] P.A.M. Dirac. (1928) "The quantum theory of the electron,"

Proc. Roy. Soc A117, 610-624.

[2] Ren, S.X. (2014) The Third Kind Of Particles.

Journal of Modern Physics, 5, 800-869.

http:/dx.doi.org/10.4236/jmp.2014.59090

[3] ShaoXu Ren. (2014) The Origins Of Spins Of Elementary Particles, ISBN 978-988-13649-7-5 
Scientific Research Publishing (SCIRP) is one of the largest Open Access journal publishers. It is currently publishing more than 200 open access, online, peer-reviewed journals covering a wide range of academic disciplines. SCIRP serves the worldwide academic communities and contributes to the progress and application of science with its publication.

Other selected journals from SCIRP are listed as below. Submit your manuscript to us via either submit@scirp.org or Online Submission Portal.
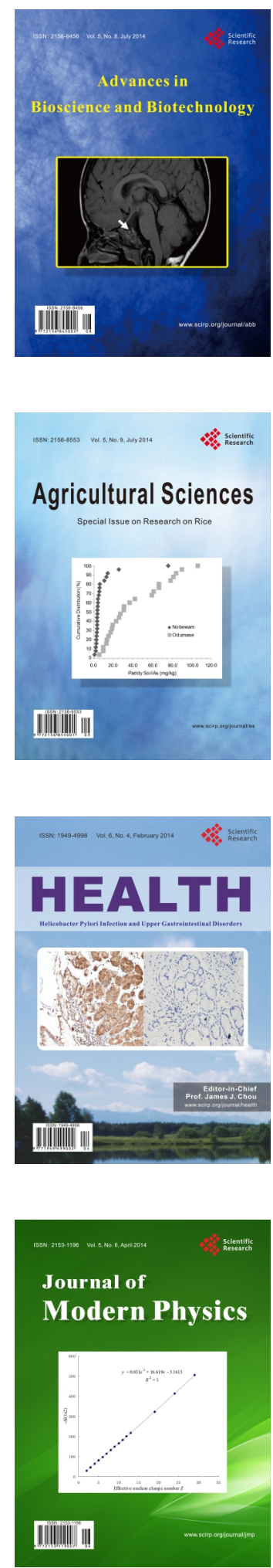
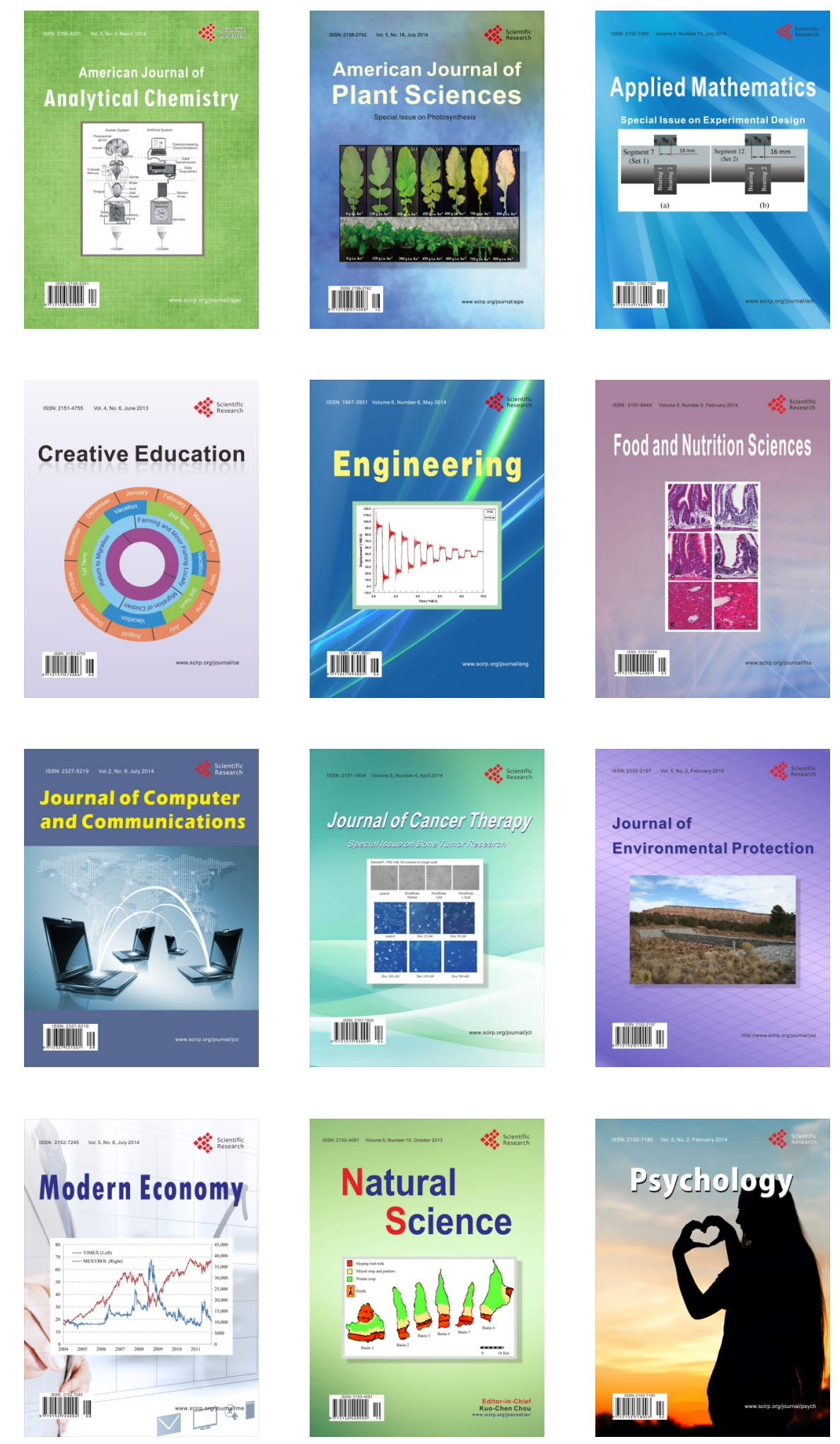